\title{
How H-bonding modifies molecular structure and pi-electron delocalization in the ring of pyridine/pyridinium derivatives involved in $\mathbf{H}$-bond complexation.
}

\author{
Tadeusz M. Krygowski ${ }^{\dagger}$, Halina Szatyłowicz ${ }^{*,+}$ and Joanna E. Zachara ${ }^{\dagger}$ \\ ${ }^{\dagger}$ Department of Chemistry, Warsaw University, Pasteura 1, 02-093 Warsaw, Poland, \\ ${ }^{t}$ Faculty of Chemistry, Warsaw University of Technology, Noakowskiego 3, 00-664 Warsaw, Poland \\ halina@chemix.ch.pw.edu.pl
}

\section{Supporting Information}

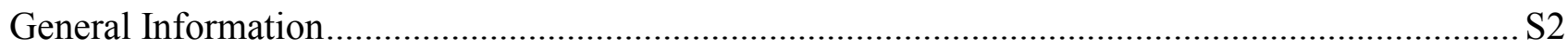

XYZ coordinates of 4-X-pyridine and 4-X-pyridinium cation .............................................. S5

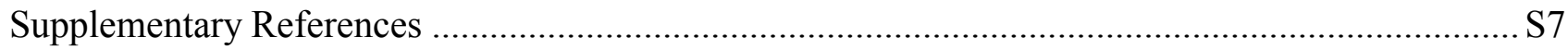




\section{General Information}

Pyridine and its derivatives play a very important role in heterocyclic chemistry. ${ }^{1}$ Hydrogen bonding is one of the most important terms used in chemistry, biochemistry and related fields. ${ }^{2}$ Both pyridine and hydrogen bond are the subject of numerous research and papers. ${ }^{3}$ The number of papers treating both topics are permanently increasing, see Figure S1. Nowadays, every hour a paper appears dealing with hydrogen bond and every four hours - a paper with the word "pyridine" included in its title or abstract or as a keyword (Figure S1). Every day a paper is published in which the terms "pyridine" (Py) and "hydrogen bond" (HB) are in the title or abstract, or as a keyword (Figure S2).

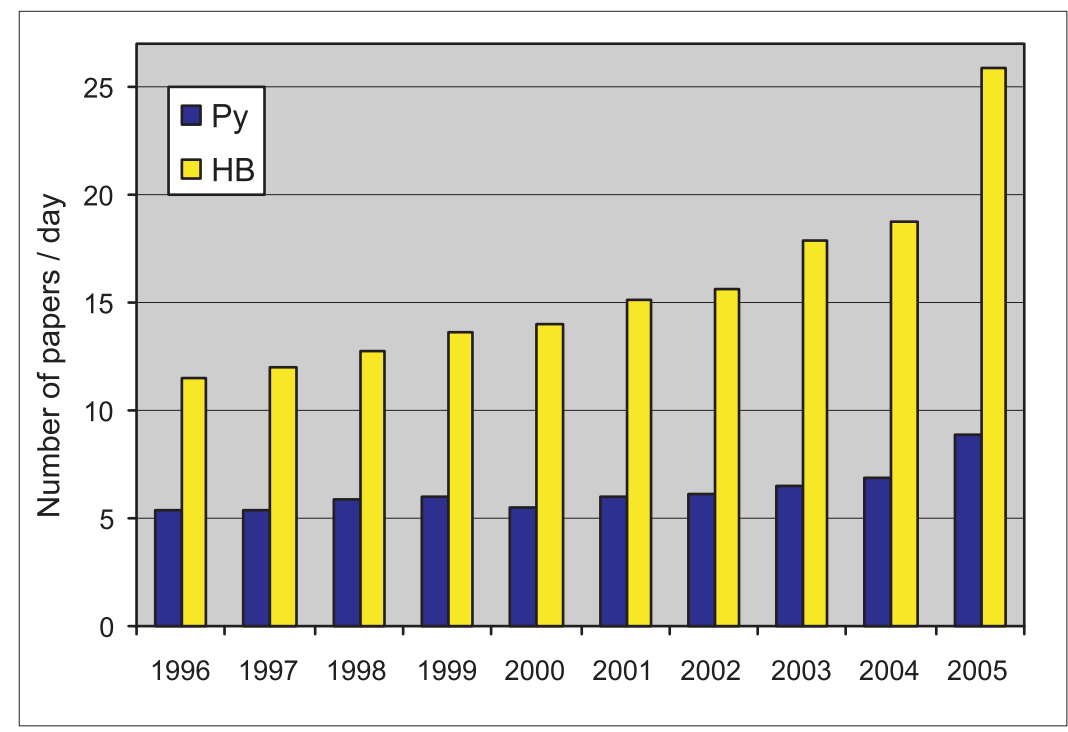

Figure S1. The number of papers in which Py (pyridine), HB (H-bonded or H-bonding or hydrogen bond*) appears in the title or abstract, or as a keyword, published every day within the last decade. ${ }^{3}$

${ }^{1}$ Pozharski, A.F.; Soldatenkov, A.T.; Katritzky, A.R. Heterocycles in Life and Society. Wiley, New York, 1997. Katritzky, A.R.; Rees, C.W.; Scriven, E.F.V., Eds, Comprehensive Heterocyclic Chemistry II. Pergamon Press, Oxford, 1996. Katritzky, A.R.; Rees, C.W., Eds, Comprehensive Heterocyclic Chemistry. Pergamon Press, Oxford, 1984.

${ }^{2}$ Jeffrey, G.A. An Introduction to Hydrogen Bonding. Oxford University Press: Oxford, 1997. Desiraju, G.R.; Steiner, T. The weak Hydrogen Bonding in Structural Chemistry and Biology. Oxford University Press, 1999.

${ }^{3}$ Institute for Scientific Information, Philadelphia (1996 - 2005). Retrieved in June 2005. 


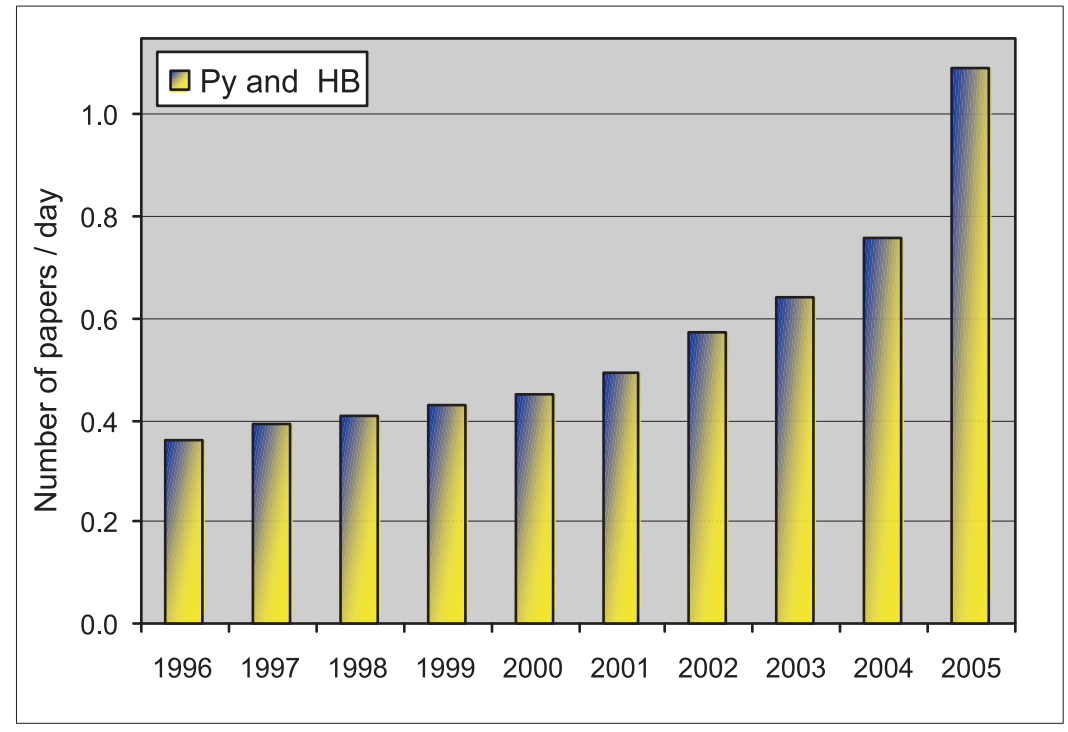

Figure S2. The number of papers in which both Py (pyridine) and HB (H-bonded or H-bonding or hydrogen bond*) appear in the title or abstract, or as a keyword, published every day within the last

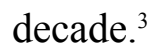

Geometries of 397 H-bonded complexes of variously substituted pyridine and pyridinium cation derivatives (287 hits) with various oxygen and nitrogen acids/bases were retrieved from $\mathrm{CSD}^{4}$ with the following restrictions: (1) the searches were performed for substituted pyridine (pyridinium cation) interacting with a nitrogen or oxygen acid (base) with an intermolecular contact between the nitrogen of pyridine (pyridinium cation) and the nearest $\mathrm{O}$ or $\mathrm{N}$ atom in acid (base) equal to or less than the sum of their van der Waals radii; ${ }^{5}$ (2) the searches were restricted to structure measurements with the reported mean estimated standard deviation (e.s.d.) of the $\mathrm{CC}$ bond $\leq 0.005 \AA$ (corresponding to AS flag $=1$ ). The data were retrieved for polysubstituted pyridine/pyridinium cation (by any of the following substituents: halogen, $-\mathrm{Me},-\mathrm{Et},-\mathrm{CHCH}_{2},-\mathrm{CN},-\mathrm{COOH},-\mathrm{COOMe},-\mathrm{COOEt},-\mathrm{COOPr},-\mathrm{COMe},-\mathrm{COPh}$, $\left.-\mathrm{CONH}_{2},-\mathrm{CF}_{3},-\mathrm{NH}_{2},-\mathrm{NMe}_{2},-\mathrm{NO}_{2},-\mathrm{OH},-\mathrm{OMe},-\mathrm{Ph},-\mathrm{SH},-\mathrm{H}\right)$ interacting with $\mathrm{N}$ or $\mathrm{O}$ atom in acid/base partner. Sometimes the molecules of the solvent were present in the crystal lattice.

\footnotetext{
${ }^{4}$ The Cambridge Structure Database, the 5.26 version, November 2004, updated February 2005.

${ }^{5}$ Bondi, A. J. Phys. Chem. 1964, 68, 441-451.
} 
A large majority of pyridine structures under study (nearly $80 \%$ hits) have been published within the last decade. The number of measurements is still increasing, as shown in Figure S3.

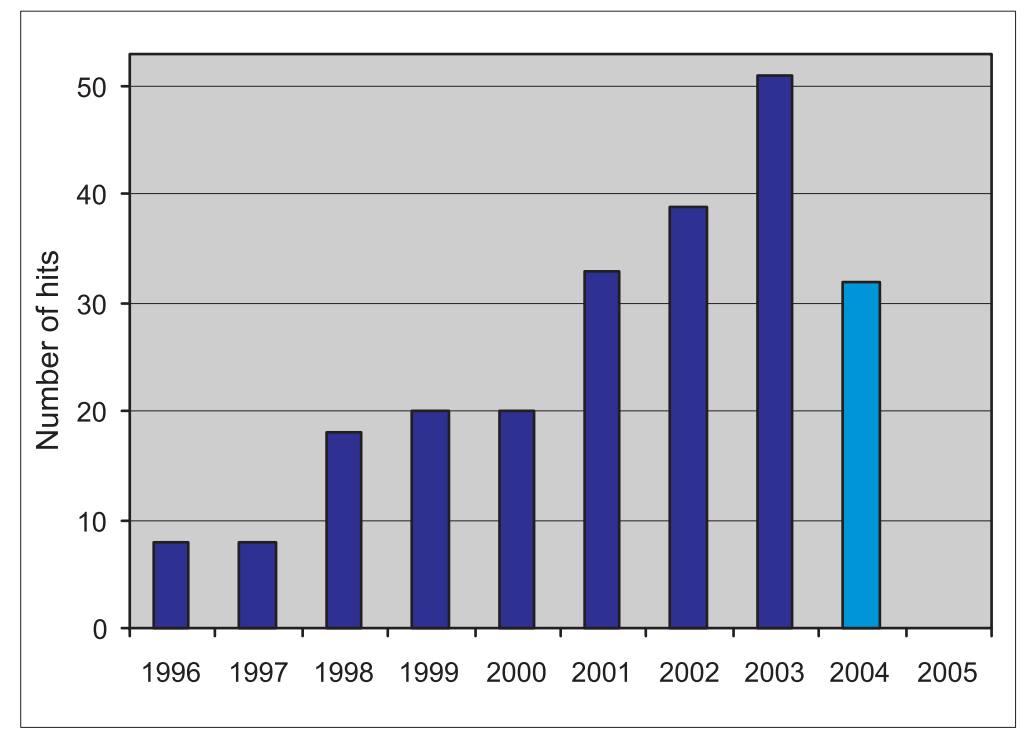

Figure S3. The number of hits pyridine under study published every year within the last decade. The Cambridge Database was updated in February 2005 therefore the data for previous year are incomplete.

B3LYP/6-311+G** level of theory was employed to optimize the molecules' geometries of 4-Xpyridine and 4-X-pyridinium cation $\left(\mathrm{X}=\mathrm{NO}_{2}, \mathrm{H}, \mathrm{OH}, \mathrm{NH}_{2}, \mathrm{O}^{-}\right)$. All ab initio calculations were performed using the Gaussian03 series of programs. The optimized molecules correspond to minima (no imaginaries frequencies). The optimized geometries are reported on pages S5 and S6. 


\section{XYZ coordinates of 4 -X-pyridine and 4-X-pyridinium cation}

\begin{tabular}{lrrr} 
4-NO2 - pyridine & \multicolumn{2}{c}{$\boldsymbol{E}=\mathbf{- 4 5 2 . 9 0 9 7 8 7}$ hartree } \\
& & & \\
N & 0.000000 & 0.000000 & 0.000000 \\
C & 0.000000 & 0.000000 & 1.335783 \\
C & 1.160201 & 0.000000 & 2.109255 \\
C & 2.367129 & 0.000000 & 1.426841 \\
C & 2.406644 & 0.000000 & 0.040692 \\
C & 1.181191 & 0.000000 & -0.624049 \\
H & 0.972899 & 0.000000 & 1.816921 \\
H & 1.130434 & 0.000000 & 3.189688 \\
N & 3.641228 & 0.000000 & 2.194284 \\
H & 3.349075 & 0.000000 & -0.488580 \\
H & 1.152091 & 0.000000 & -1.709017 \\
O & 4.682574 & 0.000000 & 1.554535 \\
O & 3.562131 & 0.000000 & 3.413924
\end{tabular}

\section{pyridine}

$\begin{array}{lr}\mathrm{N} & 0.000000 \\ \mathrm{C} & 0.000000 \\ \mathrm{C} & 1.161313 \\ \mathrm{C} & 2.391142 \\ \mathrm{C} & 2.406022 \\ \mathrm{C} & 1.187759 \\ \mathrm{H} & -0.976031 \\ \mathrm{H} & 1.097371 \\ \mathrm{H} & 3.317383 \\ \mathrm{H} & 3.338100 \\ \mathrm{H} & 1.163649\end{array}$

0.000000

0.000000

0.000000

0.000000

0.000000

0.000000

0.000000

0.000000

0.000000

0.000000

0.000000
0.000000

1.336490

2.108187

1.456215

0.064052

$-0.613544$

1.813877

3.189991

2.020045

$-0.488791$

$-1.699753$

4-NO2-pyridinium

$\begin{array}{lr}\mathrm{N} & 0.000000 \\ \mathrm{C} & 0.000000 \\ \mathrm{C} & 1.204452 \\ \mathrm{C} & 2.368917 \\ \mathrm{C} & 2.356172 \\ \mathrm{C} & 1.125514 \\ \mathrm{H} & -0.963675 \\ \mathrm{H} & 1.242708 \\ \mathrm{~N} & 3.695551 \\ \mathrm{H} & 3.278888 \\ \mathrm{H} & 1.002771 \\ \mathrm{H} & -0.896362 \\ \mathrm{O} & 4.681446 \\ \mathrm{O} & 3.649414\end{array}$

\section{pyridinium}

$\begin{array}{lr}\mathrm{N} & 0.000000 \\ \mathrm{C} & 0.000000 \\ \mathrm{C} & 1.204546 \\ \mathrm{C} & 2.393178 \\ \mathrm{C} & 2.354903 \\ \mathrm{C} & 1.130151 \\ \mathrm{H} & -0.965429 \\ \mathrm{H} & 1.207812 \\ \mathrm{H} & 3.346493 \\ \mathrm{H} & 3.261810 \\ \mathrm{H} & 1.009500 \\ \mathrm{H} & -0.893931\end{array}$

$E=-453.253666$ hartee

0.000000

0.000000

0.000000

0.000000

0.000000

0.000000

0.000000

0.000000

0.000000

0.000000

0.000000

0.000000

0.000000

0.000000

0.000000
1.350309

2.028531

1.271621

$-0.117242$

$-0.746347$

1.842150

3.109972

1.983649

$-0.682582$

$-1.821282$

$-0.481284$

1.274524

3.197219

\section{4-OH-pyridine}

$\begin{array}{lr}\mathrm{N} & 0.000000 \\ \mathrm{C} & 0.000000 \\ \mathrm{C} & 1.147258 \\ \mathrm{C} & 2.383995 \\ \mathrm{C} & 2.409047 \\ \mathrm{C} & 1.194281 \\ \mathrm{H} & -0.975990 \\ \mathrm{H} & 1.095124 \\ \mathrm{O} & 3.506616 \\ \mathrm{H} & 3.346658 \\ \mathrm{H} & 1.187846 \\ \mathrm{H} & 4.290368\end{array}$

$E=-323.601551$ hartree

0.000000

0.000000

0.000000

0.000000

0.000000

0.000000

0.000000

0.000000

0.000000

0.000000

0.000000

0.000000
0.000000

1.338801

1.478503

0.083342

$-0.597408$

1.815740

3.204546

2.245923

$-0.463650$

$-1.683861$

1.685295
2.123104

\section{4-OH-pyridinium}

$\begin{array}{lr}\mathrm{N} & 0.000000 \\ \mathrm{C} & 0.000000 \\ \mathrm{C} & 1.185588 \\ \mathrm{C} & 2.397248 \\ \mathrm{C} & 2.360925 \\ \mathrm{C} & 1.148821 \\ \mathrm{H} & -0.965257 \\ \mathrm{H} & 1.171173 \\ \mathrm{O} & 3.592637 \\ \mathrm{H} & 3.283248 \\ \mathrm{H} & 1.047028 \\ \mathrm{H} & -0.887691 \\ \mathrm{H} & 3.558242\end{array}$

$E=-248.719068$ hartree

0.000000

0.000000

0.000000

0.000000

0.000000

0.000000

0.000000

0.000000

0.000000

0.000000

0.000000

0.000000
0.000000

1.350823

2.026086

1.293609

$-0.102073$

$-0.739940$

1.838729

3.107945

1.808899

$-0.691941$

$-1.814904$

$-0.483210$
$E=-323.975952$ hartree

0.000000

0.000000

0.000000

0.000000

0.000000

0.000000

0.000000

0.000000

0.000000

0.000000

0.000000

0.000000

0.000000
0.000000

1.352747

2.043454

1.323556

$-0.085748$

$-0.721837$

1.840978

3.126434

1.890286

$-0.650907$

$-1.798631$

$-0.490449$

2.857300 
Supporting Information for The Journal of Organic Chemistry

Krygowski, T M.; Szatylowicz, H., Zachara J.E

HowH-bonding modifies molecular structure.

\section{4 -NH2 -pyridine}

$\begin{array}{lr}\mathrm{N} & 0.000000 \\ \mathrm{C} & 0.000000 \\ \mathrm{C} & 1.144558 \\ \mathrm{C} & 2.400351 \\ \mathrm{C} & 2.411320 \\ \mathrm{C} & 1.202211 \\ \mathrm{H} & -0.976595 \\ \mathrm{H} & 1.063618 \\ \mathrm{~N} & 3.572542 \\ \mathrm{H} & 3.347758 \\ \mathrm{H} & 1.201884 \\ \mathrm{H} & 4.422123 \\ \mathrm{H} & 3.526286\end{array}$

$\begin{array}{rr}E=-303.732400 & \text { hartree } \\ 0.000000 & 0.000000 \\ 0.000000 & 1.338215 \\ 0.000000 & 2.124535 \\ -0.000068 & 1.498487 \\ -0.001721 & 0.095346 \\ -0.001272 & -0.587642 \\ 0.001358 & 1.815111 \\ -0.003650 & 3.206378 \\ -0.047601 & 2.230210 \\ -0.006755 & -0.452371 \\ -0.001072 & -1.674469 \\ 0.229503 & 1.763937 \\ 0.227745 & 3.198769\end{array}$

\section{4-NH2-pyridinium $E=-304.121447$ hartree}

$\begin{array}{rrrr}\mathrm{N} & 1.785908 & 0.000144 & -0.023371 \\ \mathrm{C} & 1.121554 & 1.184460 & 0.001057 \\ \mathrm{C} & -0.242893 & 1.215983 & 0.025242 \\ \mathrm{C} & -0.980448 & 0.000009 & 0.010512 \\ \mathrm{C} & -0.242531 & -1.215960 & 0.025571 \\ \mathrm{C} & 1.121826 & -1.184432 & 0.001024 \\ \mathrm{H} & 1.727458 & 2.080338 & -0.003668 \\ \mathrm{H} & -0.749899 & 2.172186 & 0.040097 \\ \mathrm{~N} & -2.318304 & -0.000173 & -0.025318 \\ \mathrm{H} & -0.749506 & -2.172167 & 0.040407 \\ \mathrm{H} & 1.728093 & -2.080072 & -0.004344 \\ \mathrm{H} & 2.798476 & 0.000116 & -0.048379 \\ \mathrm{H} & -2.846261 & -0.859783 & -0.039053 \\ \mathrm{H} & -2.846626 & 0.859223 & -0.037378\end{array}$

\section{4-o (-) -pyridine}

$\begin{array}{lr}\mathrm{N} & 0.000000 \\ \mathrm{C} & 0.000000 \\ \mathrm{C} & 1.122101 \\ \mathrm{C} & 2.453022 \\ \mathrm{C} & 2.426929 \\ \mathrm{C} & 1.232977 \\ \mathrm{H} & -0.988173 \\ \mathrm{H} & 1.022753 \\ \mathrm{O} & 3.511839 \\ \mathrm{H} & 3.374780 \\ \mathrm{H} & 1.254453\end{array}$

$\begin{array}{lr}E=-323.053159 & \text { hartree } \\ 0.000000 & 0.000000 \\ 0.000000 & 1.349837 \\ 0.000000 & 2.156557 \\ 0.000000 & 1.591614 \\ 0.000000 & 0.145803 \\ 0.000000 & -0.550112 \\ 0.000000 & 1.814649 \\ 0.000000 & 3.238985 \\ 0.000000 & 2.278778 \\ 0.000000 & -0.386301 \\ 0.000000 & -1.641841\end{array}$

$\begin{array}{crrr}\text { 4-O (-) pyridinium } & E=-\mathbf{3 2 3 . 5 9 9 7 8 5} \text { hartree } \\ \text { N } & 0.000000 & 0.000000 & 0.000000 \\ \mathrm{C} & 0.000000 & 0.000000 & 1.372899 \\ \mathrm{C} & 1.158318 & 0.000000 & 2.074091 \\ \mathrm{C} & 2.462049 & 0.000000 & 1.405824 \\ \mathrm{C} & 2.375104 & 0.000000 & -0.056739 \\ \mathrm{C} & 1.182614 & 0.000000 & -0.697989 \\ \mathrm{H} & -0.976169 & 0.000000 & 1.841936 \\ \mathrm{H} & 1.139611 & 0.000000 & 3.156345 \\ \mathrm{O} & 3.531735 & 0.000000 & 2.016454 \\ \mathrm{H} & 3.297756 & 0.000000 & -0.622799 \\ \mathrm{H} & 1.090086 & 0.000000 & -1.776956 \\ \mathrm{H} & -0.874236 & 0.000000 & -0.499361\end{array}$




\section{Supplementary references}

ACESIL Quinol pyridine; C6 H6 O2,C5 H5 N1; I.D.H.Oswald, W.D.S.Motherwell, S.Parsons; Acta Crystallogr.,Sect.E: Struct.Rep.Online , 60, o1967, 2004.

ACEWIP bis(Trimethylpyridinium) chloranilate; 2(C8 H12 N1 1+),C6 Cl2 O4 2-; H.Ishida; Acta Crystallogr.,Sect.E: Struct.Rep.Online , 60, o2005, 2004.

AFEDIY 4-Aminopyridinium 2-chloro-4-nitrobenzoate; C5 H7 N2 1+,C7 H3 C11 N1 O4 1-;

T.Sugiyama, J.Meng, T.Matsuura; Acta Crystallogr.,Sect.C: Cryst.Struct.Commun., 58, o242, 2002.

AFEDOE 4-Aminopyridinium 2-chloro-5-nitrobenzoate; C5 H7 N2 1+,C7 H3 Cl1 N1 O4 1-; T.Sugiyama, J.Meng, T.Matsuura; Acta Crystallogr.,Sect.C: Cryst.Struct.Commun., 58, o242, 2002.

AFEDUK 4-Aminopyridinium 5-chloro-2-nitrobenzoate; C5 H7 N2 1+,C7 H3 C11 N1 O4 1-; T.Sugiyama, J.Meng, T.Matsuura; Acta Crystallogr.,Sect.C: Cryst.Struct.Commun., 58, o242, 2002.

AFEFAS 4-Aminopyridinium 4-chloro-2-nitrobenzoate; C5 H7 N2 1+,C7 H3 Cl1 N1 O4 1-; T.Sugiyama, J.Meng, T.Matsuura; Acta Crystallogr.,Sect.C: Cryst.Struct.Commun., 58, o242, 2002.

AGOYIE Triphenylsilanol 4-phenylpyridine; C18 H16 O1 Sil,C11 H9 N1; A.E.Goeta, S.E.Lawrence, M.M.Meehan, A.O'Dowd, T.R.Spalding; Polyhedron , 21, 1689, 2002.

AJAKAX Isonicotinamide hex-2-enoic acid; C6 H6 N2 O1,C6 H10 O2; C.B.Aakeroy, A.M.Beatty, B.A.Helfrich, M.Nieuwenhuyzen; Cryst.Growth Des., 3, 159, 2003.

AJAKEB Isonicotinamide 4-nitrobenzoic acid; C6 H6 N2 O1,C7 H5 N1 O4; C.B.Aakeroy, A.M.Beatty, B.A.Helfrich, M.Nieuwenhuyzen; Cryst.Growth Des., 3, 159, 2003.

AJAKIF Isonicotinamide 3,5-dinitrobenzoic acid 4-methylbenzoic acid; C6 H6 N2 O1,C7 H4 N2

O6,C8 H8 O2; C.B.Aakeroy, A.M.Beatty, B.A.Helfrich, M.Nieuwenhuyzen; Cryst.Growth Des., 3, 159, 2003.

AJAKOL 2-Amino-5-nitropyrimidine 2-amino-3-nitropyridine; C5 H5 N3 O2,C4 H4 N4 O2;

C.B.Aakeroy, A.M.Beatty, B.A.Helfrich, M.Nieuwenhuyzen; Cryst.Growth Des., 3, 159, 2003.

AJEBIA Pyridinium 3,5-dinitrosalicylate 3,5-dinitrosalicylic acid; C5 H6 N1 1+,C7 H3 N2 O7 1-

,C7 H4 N2 O7; G.Smith, U.D.Wermuth, P.C.Healy, J.M.White; Aust.J.Chem., 56, 707, 2003.

AJEBOG 4-Cyanopyridinium 3,5-dinitrosalicylate; C6 H5 N2 1+,C7 H3 N2 O7 1-; G.Smith, U.D.Wermuth, P.C.Healy, J.M.White; Aust.J.Chem., 56, 707, 2003. 
AJULEW 4-Dimethylaminopyridinium picrate; C7 H11 N2 1+,C6 H2 N3 O7 1-; N.Vembu, M.Nallu, J.Garrison, W.J.Youngs; Acta Crystallogr.,Sect.E: Struct.Rep.Online , 59, o913, 2003.

AMCLPY 2-Amino-5-chloropyridine; C5 H5 C11 N2; A.Kvick, M.Backeus; Acta Crystallogr.,Sect.B: Struct.Crystallogr.Cryst.Chem., 30, 474, 1974.

AMCLPY11 2-Amino-5-chloropyridine; C5 H5 C11 N2; A.Kvick, R.Thomas, T.F.Koetzle; Acta Crystallogr.,Sect.B: Struct.Crystallogr.Cryst.Chem., 32, 224, 1976.

AMEPYD 2-Amino-4-methylpyridine; C6 H8 N2; A.Kvick, J.Noordik; Acta Crystallogr.,Sect.B: Struct.Crystallogr.Cryst.Chem., 33, 2862, 1977.

AMNTPY 2-Amino-3-nitropyridine; C5 H5 N3 O2; R.Destro, T.Pilati, M.Simonetta; Acta Crystallogr.,Sect.B: Struct.Crystallogr.Cryst.Chem., 31, 2883, 1975.

AMNTPY01 2-Amino-3-nitropyridine; C5 H5 N3 O2; C.B.Aakeroy, A.M.Beatty, M.Nieuwenhuyzen, Min Zou; J.Mater.Chem., 8, 1385, 1998.

AMPYRD 2-Aminopyridine; C5 H6 N2; M.Chao, E.Schempp, R.D.Rosenstein; Acta Crystallogr.,Sect.B: Struct.Crystallogr.Cryst.Chem., 31, 2922, 1975.

AMPYRE 4-Aminopyridine; C5 H6 N2; M.Chao, E.Schempp; Acta Crystallogr.,Sect.B: Struct.Crystallogr.Cryst.Chem., 33, 1557, 1977.

ASAXOH 3-Nitrobenzoic acid isonicotinamide; C6 H6 N2 O1,C7 H5 N1 O4; C.B.Aakeroy, J.Desper, B.A.Helfrich; CrystEngComm , 6, 19, 2004.

ASAXUN Isonicotinamide 4-fluorobenzoic acid; C6 H6 N2 O1,C7 H5 F1 O2; C.B.Aakeroy, J.Desper, B.A.Helfrich; CrystEngComm , 6, 19, 2004.

AWUDEB 3,5-Dinitrobenzoic acid nicotinic acid; C7 H4 N2 O6,C6 H5 N1 O2; Jun Zhu, Ji-Min Zheng; Jiegou Huaxue(Chin.)(Chinese J.Struct.Chem.) , 23, 417, 2004.

BAJTEM 2-bromo-5-pyridylboronic acid; C5 H5 B1 Br1 N1 O2; P.R.Parry, Changsheng Wang, A.S.Batsanov, M.R.Bryce, B.Tarbit; J.Org.Chem., 67, 7541, 2002.

BAJTEM01 2-bromo-5-pyridylboronic acid; C5 H5 B1 Br1 N1 O2; P.R.Parry, Changsheng Wang, A.S.Batsanov, M.R.Bryce, B.Tarbit; J.Org.Chem., 67, 7541, 2002.

BAJTEM02 (6-Bromopyridin-3-yl)boronic acid; C5 H5 B1 Br1 N1 O2; J.Sopkova-de Oliveira Santos, J.-C.Lancelot, A.Bouillon, S.Rault; Acta Crystallogr.,Sect.C: Cryst.Struct.Commun., 59, o111, 2003.

BAJTIQ 2-chloro-5-pyridylboronic acid; C5 H5 B1 C11 N1 O2; P.R.Parry, Changsheng Wang, A.S.Batsanov, M.R.Bryce, B.Tarbit; J.Org.Chem., 67, 7541, 2002.

BARAPY10 5,5-Diethylbarbituric acid bis(2-aminopyridine); C8 H12 N2 O3,2(C5 H6 N2); I.-N.Hsu, B.M.Craven; Acta Crystallogr.,Sect.B: Struct.Crystallogr.Cryst.Chem., 30, 994, 1974. 
BAXZAC tris(3-Aminopyridinium) benzene-1,3,5-tricarboxylate-2,4,6-tricarboxylic acid; 3(C5 H7

N2 1+),C12 H3 O12 3-; N.Kobayashi, T.Naito, T.Inabe; Bull.Chem.Soc.Jpn., 76, 1351, 2003.

BAXZEG bis(4-Methylpyridinium) bis(4-methylpyridine) benzene-1,4-dicarboxylate-2,3,5,6tetracarboxylic acid; 2(C6 H7 N1),2(C6 H8 N1 1+),C12 H4 O12 2-; N.Kobayashi, T.Naito, T.Inabe; Bull.Chem.Soc.Jpn., 76, 1351, 2003.

BAXZOQ bis(Pyridinium) benzene-1,2-dicarboxylate-3,4,5,6-tetracarboxylic acid dihydrate; 2(C5 H6 N1 1+),C12 H4 O12 2-,2(H2 O1); N.Kobayashi, T.Naito, T.Inabe; Bull.Chem.Soc.Jpn., 76, 1351, 2003.

BAXZUW bis(3-Methylpyridinium) benzene-1,3-dicarboxylate-2,4,5,6-tetracarboxylic acid; 2(C6 H8 N1 1+),C12 H4 O12 2-; N.Kobayashi, T.Naito, T.Inabe; Bull.Chem.Soc.Jpn., 76, 1351, 2003. BEJZUL 5-Hydroxy-4-(methoxycarbonyl)pyridinium-3-carboxylate; C8 H7 N1 O5; A.Damsgaard, R.Hazell, M.Bols; Acta Chem.Scand., 53, 521, 1999.

BERCEH 1,3-Di(9-anthryl)propan-2-ol pyridine clathrate; C31 H24 O1,C5 H5 N1; N.Hayashi, T.Mori, K.Yamaguchi, N.Tsukamoto, K.Matsumoto; Heterocycles , 61, 357, 2003.

BEXPUQ 2-Aminopyridinium 3-aminobenzoate; C5 H7 N2 1+,C7 H6 N1 O2 1-; S.Banerjee, R.Murugavel; Cryst.Growth Des., 4, 545, 2004.

BIDSUD 3-Methoxy-2-phenyl-6-(trifluoromethyl)-4-pyridinol; C13 H10 F3 N1 O2; O.Flogel, J.Dash, I.Brudgam, H.Hartl, H.-U.Reissig; Chem.-Eur.J., 10, 4283, 2004.

BIRYIK10 3-Hydroxypyridine; C5 H5 N1 O1; U.Ohms, H.Guth, W.Treutmann; Z.Kristallogr.,Kristallgeom.,Kristallphys.,Kristallchem., 162, 299, 1983.

BRHXPY 6-Bromo-3-hydroxypyridine; C5 H4 Br1 N1 O1; A.Kvick; Acta Crystallogr.,Sect.B: Struct.Crystallogr.Cryst.Chem., 32, 220, 1976.

BUDWEC Benzoic acid isonicotinamide; C7 H6 O2,C6 H6 N2 O1; C.B.Aakeroy, A.M.Beatty, B.A.Helfrich; Angew.Chem.,Int.Ed., 40, 3240, 2001.

BUDZUV 3,5-Dinitrobenzoic acid isonicotinamide 3-methylbenzoic acid; C7 H4 N2 O6,C6 H6 N2

O1,C8 H8 O2; C.B.Aakeroy, A.M.Beatty, B.A.Helfrich; Angew.Chem.,Int.Ed., 40, 3240, 2001.

BUFBIP 3,5-Dinitrobenzoic acid isonicotinamide 4-(N,N-dimethylamino)benzoic acid; C7 H4 N2

O6,C6 H6 N2 O1,C9 H11 N1 O2; C.B.Aakeroy, A.M.Beatty, B.A.Helfrich; Angew.Chem.,Int.Ed., 40, 3240, 2001.

BUFQAU 3,5-Dinitrobenzoic acid isonicotinamide 4-hydroxy-3-methoxycinnamic acid; C7 H4 N2 O6,C6 H6 N2 O1,C10 H10 O4; C.B.Aakeroy, A.M.Beatty, B.A.Helfrich; Angew.Chem.,Int.Ed., 40, 3240, 2001.

CAXNOE 4-Nitrophenol 4-methylpyridine; C6 H5 N1 O3,C6 H7 N1; Zhi Min Jin, Yuan Jiang Pan, Duan Jun Xu, Yuan Zhi Xu; Acta Crystallogr.,Sect.C: Cryst.Struct.Commun., 56, e69, 2000. 
CEPPIW Pyridinium bis(2,3-naphthalenediolato)-phenylsilicate(iv); C26 H17 O4 Si1 1-,C5 H6 N1

1+; R.R.Holmes, R.O.Day, J.J.Harland, J.M.Holmes; Organometallics , 3, 347, 1984.

CETPAS Pyridinium pyridinium-2,6-bis(carbothioate); C7 H4 N1 O2 S2 1-,C5 H6 N1 1+;

U.Hildebrand, W.Ockerls, J.Lex, H.Budzikiewicz; Phosphorus and Sulfur , 16, 361, 1983.

CHPYRD 6-Chloro-2-hydroxypyridine; C5 H4 Cl1 N1 O1; A.Kvick, I.Olovsson; Ark.Kemi , 30, 71, 1969.

CINMER Pyridine 3,4-dicarboxylic acid; C7 H5 N1 O4; F.Takusagawa, K.Hirotsu, A.Shimada; Bull.Chem.Soc.Jpn., 46, 2669, 1973.

CUMQAC 1,1,6,6-Tetraphenylhexa-2,4-diyne-1,6-diol bis(2,4-lutidine); C30 H22 O2,2(C7 H9 N1);

M.R.Caira, L.R.Nassimbeni, F.Toda, D.Vujovic; J.Chem.Soc.,Perkin Trans.2 , , 2681, 1999.

CUMQEG 1,1,6,6-Tetraphenylhexa-2,4-diyne-1,6-diol bis(3,5-lutidine); C30 H22 O2,2(C7 H9 N1);

M.R.Caira, L.R.Nassimbeni, F.Toda, D.Vujovic; J.Chem.Soc.,Perkin Trans.2 , , 2681, 1999.

CUMQIK 1,1,6,6-Tetraphenylhexa-2,4-diyne-1,6-diol bis(2,6-lutidine); C30 H22 O2,2(C7 H9 N1);

M.R.Caira, L.R.Nassimbeni, F.Toda, D.Vujovic; J.Chem.Soc.,Perkin Trans.2 , , 2681, 1999.

DEFCUM bis(Pyridinium) oxalate oxalic acid; 2(C5 H6 N1 1+),C2 O4 2-,C2 H2 O4;

G.R.Newkome, K.J.Theriot, F.R.Fronczek; Acta Crystallogr.,Sect.C: Cryst.Struct.Commun., 41, $1642,1985$.

DMAPYC 4-Dimethylaminopyridine hydrochloride dihydrate; C7 H11 N2 1+,Cl1 1-,2(H2 O1);

M.Chao, E.Schempp, R.D.Rosenstein; Acta Crystallogr.,Sect.B: Struct.Crystallogr.Cryst.Chem., 33, 1820, 1977.

DOCWUN 1-(1,2-bis(Hydroxyimino)propyl)-3-methyl-2-pyridone 2-amino-3-methylpyridine; C9 H11 N3 O3,C6 H8 N2; A.A.Espenbetov, Yu.T.Struchkov, I.A.Poplavskaya, R.G.Kurmangalieva; Izv.Akad.Nauk Kaz.SSR,Ser.Khim.(Russ.)(Bull.Acad.Sci.Kazakhstan,Chem.) , , 57-6, 1985.

DOQSUX01 2,3-Dihydroxypyridine; C5 H5 N1 O2; T.V.Vovk, O.V.Koval'chukova, B.E.Zaitsev, S.B.Strashnova, V.K.Belsky, A.I.Stash; Koord.Khim.(Russ.)(Coord.Chem.), 29, 312, 2003.

DUVLUB Dipyridinium bis(hydrogen-oxalate) oxalic acid; 2(C5 H6 N1 1+),2(C2 H1 O4 1-),C2 H2 O4; G.R.Newkome, K.J.Theriot, F.R.Fronczek; Acta Crystallogr.,Sect.C: Cryst.Struct.Commun., 42, 1539, 1986.

EBOKIP 2,6-Diaminopyridinium 2,4,6-trinitrobenzoate; C5 H8 N3 1+,C7 H2 N3 O8 1-; G.Smith,

R.C.Bott, A.D.Rae, A.C.Willis; Aust.J.Chem., 53, 531, 2000.

EDADOC 2-Amino-5-nitropyridinium 2-methyl-5-nitrobenzenesulfonate; C5 H6 N3 O2 1+,C7 H6

N1 O5 S1 1-; H.Koshima, M.Hamada, I.Yagi, K.Uosaki; Cryst.Growth Des., 1, 467, 2001.

EDAFEU 2-Amino-5-nitropyridinium 4-chlorophenylsulfonate; C5 H6 N3 O2 1+,C6 H5 O4 S1 1-; H.Koshima, M.Hamada, I.Yagi, K.Uosaki; Cryst.Growth Des., 1, 467, 2001. 
EFAWAJ 6-aminopyridine-3-thiol; C5 H6 N2 S1; J.R.Sabino, C.H.T.P.da Silva, M.Yonashiro; Acta Crystallogr.,Sect.C: Cryst.Struct.Commun., 58, o78, 2002.

EHOWIH02 Isonicotinamide; C6 H6 N2 O1; C.B.Aakeroy, A.M.Beatty, B.A.Helfrich, M.Nieuwenhuyzen; Cryst.Growth Des., 3, 159, 2003.

EKEPEP 4-Aminopyridinium hydrogen maleate; C4 H3 O4 1-,C5 H7 N2 1+; N.Lah, I.Leban; Acta Crystallogr.,Sect.C: Cryst.Struct.Commun., 59, o537, 2003.

ELEWOH 4-Dimethylaminopyridinium 2,4-dinitrophenolate; C7 H11 N2 1+,C6 H3 N2 O5 1-; N.Vembu, M.Nallu, E.C.Spencer, J.A.K.Howard; Acta Crystallogr.,Sect.E: Struct.Rep.Online , 59, o1383, 2003.

EMINUJ Dinicotinamidium squarate; 2(C6 H7 N2 O1 1+),C4 O4 2-; A.Bulut, O.Z.Yesilel, N.Dege, H.Icbudak, H.Olmez, O.Buyukgungor; Acta Crystallogr.,Sect.C: Cryst.Struct.Commun., 59, o727, 2003.

EMOXEJ Pyridinium narcistatin sesquihydrate; C14 H11 N1 O9 P1 1-,C5 H6 N1 1+,1.5(H2 O1); G.R.Pettit, N.Melody, M.Simpson, M.Thompson, D.L.Herald, J.C.Knight; J.Nat.Prod., 66, 92, 2003.

EMUBAP 2,8,14,20-tetraethyl-4,6,10,12,16,18,22,24-octahydroxycalix(4)arene pyridine clathrate ethanol solvate; C36 H40 O8,C5 H5 N1,3(C2 H6 O1); M.Nissinen, K.Rissanen; Supramol.Chem., 15, 581, 2003.

ENEKAJ 4-Dimethylaminopyridinium (4-chlorobenzoyl)-cyano-(6-chloro-7-methyl-1,1-dioxo1,4,2-benzodithiazin-3-yl)methanide; C7 H11 N2 1+,C17 H9 Cl2 N2 O3 S2 1-; Z.Brzozowski, F.Saczewski, M.Gdaniec; Eur.J.Med.Chem., 38, 991, 2003.

ERAVEY (Hydrogen nicotinic acid) nicotinic acid iodide; C6 H6 N1 O2 1+,C6 H5 N1 O2,I1 1-; M.A.S.Goher, F.A.Mautner, T.C.W.Mak, M.A.M.Abu-Youssef; Monatsh.Chem., 134, 1519, 2003.

ESAQIY 2,6-Dimethylpyridinium di(methanesulfanyl)amide; C5 H8 N3 1+,C2 H6 N1 O4 S2 1-;

K.Wijaya, O.Moers, A.Blaschette, P.G.Jones; Z.Naturforsch.,BChem.Sci., 59, 17, 2004.

EWOVIV 4-Phenylpyridinium hydrogensquarate; C11 H10 N1 1+,C4 H1 O4 1-; T.Kolev, R.Wortmann, M.Spiteller, W.S.Sheldrick, M.Heller; Acta Crystallogr.,Sect.E: Struct.Rep.Online , 60, o956, 2004.

EXASAX 2,7-bis-(3-Hydroxy-3,3-diphenyl-prop-1-ynyl)-fluoren-9-one bis(pyridine) clathrate; C43 H28 O3,2(C5 H5 N1); J.L.Scott, T.Yamada, K.Tanaka; New J.Chem.(Nouv.J.Chim.) , 28, 447, 2004. 
EXASEB 2,7-bis-(3-Hydroxy-3,3-diphenyl-prop-1-ynyl)-fluoren-9-one 3-acetylpyridine clathrate; C43 H28 O3,C7 H7 N1 O1; J.L.Scott, T.Yamada, K.Tanaka; New J.Chem.(Nouv.J.Chim.) , 28, 447,2004

EYIXAL Picolinamidium squarate; C6 H7 N2 O1 1+,C4 H1 O4 1-; I.Ucar, A.Bulut, O.Z.Yesilel, O.Buyukgungor; Acta Crystallogr.,Sect.C: Cryst.Struct.Commun., 60, o585, 2004.

FOYLEK 2,6-Diaminopyridine; C5 H7 N3; C.H.Schwalbe, G.J.B.Williams, T.F.Koetzle; Acta Crystallogr.,Sect.C: Cryst.Struct.Commun., 43, 2191, 1987.

GADGUN02 4-Methylpyridine pentachlorophenol; C6 H7 N1,C6 H1 Cl5 O1; Z.Malarski, I.Majerz, T.Lis; J.Mol.Struct., 380, 249, 1996.

GADGUN03 4-Methylpyridine pentachlorophenol; C6 H1 Cl5 O1,C6 H7 N1; T.Steiner, I.Majerz, C.C.Wilson; Angew.Chem.,Int.Ed., 40, 2651, 2001.

GADGUN04 4-Methylpyridine pentachlorophenol; C6 H1 Cl5 O1,C6 H7 N1; T.Steiner, I.Majerz, C.C.Wilson; Angew.Chem.,Int.Ed., 40, 2651, 2001.

GADGUN05 4-Methylpyridine pentachlorophenol; C6 H1 Cl5 O1,C6 H7 N1; T.Steiner, I.Majerz, C.C.Wilson; Angew.Chem.,Int.Ed., 40, 2651, 2001.

GIVYAL 2-Amino-5-nitropyridine monohydrate; C5 H5 N3 O2,H2 O1; C.B.Aakeroy, A.M.Beatty, M.Nieuwenhuyzen, Min Zou; J.Mater.Chem., 8, 1385, 1998.

GODNAO 2,4,6-Trimethylpyridine benzoic acid; C8 H11 N1,C7 H6 O2; C.Foces-Foces, A.L.Llamas-Saiz, P.Lorente, N.S.Golubev, H.-H.Limbach; Acta Crystallogr.,Sect.C: Cryst.Struct.Commun., 55, 377, 1999.

GODNES 2,4,6-Trimethylpyridinium 2-nitrobenzoate; C8 H12 N1 1+,C7 H4 N1 O4 1-; C.Foces-

Foces, A.L.Llamas-Saiz, P.Lorente, N.S.Golubev, H.-H.Limbach; Acta Crystallogr.,Sect.C: Cryst.Struct.Commun., 55, 377, 1999.

GODNIW 2,4,6-Trimethylpyridinium 3,5-dinitrobenzoate; C8 H12 N1 1+,C7 H3 N2 O6 1-; C.Foces-Foces, A.L.Llamas-Saiz, P.Lorente, N.S.Golubev, H.-H.Limbach; Acta Crystallogr.,Sect.C: Cryst.Struct.Commun., 55, 377, 1999.

GOHHOA bis(2-Pyridone)hydrogen dimethylsulfonamide; C5 H5 N1 O1,C5 H6 N1 O1 1+,C2 H6 N1 O4 S2 1-; K.Wijaya, O.Moers, A.Blaschette, P.G.Jones; Z.Naturforsch.,BChem.Sci., 54, 643, 1999.

GOHHUG Hydrogen bis(6-methyl-2-pyridone) dimethylsulfonamide; C6 H8 N1 O1 1+,C6 H7 N1 O1,C2 H6 N1 O4 S2 1-; K.Wijaya, O.Moers, A.Blaschette, P.G.Jones; Z.Naturforsch.,BChem.Sci., 54, 643, 1999. 
GOMBEP Pyridinium 2,5-dichloro-6-hydroxy-p-benzoquinone-3-olate monohydrate; C5 H6 N1 1+,C6 $\quad \mathrm{H} 1 \quad \mathrm{Cl} 2 \quad \mathrm{O} 4 \quad$ 1-,H2 O1; H.Ishida, S.Kashino; Acta Crystallogr.,Sect.C: Cryst.Struct.Commun., 55, 1149, 1999.

GOWVAP N,N'-Di(4-nitrobenzosulfuryl)-p-phenylenediamine tetrapyridine clathrate; C18 H14 N4 O8 S2,4(C5 H5 N1); H.Bock, N.Nagel, P.Eller; Z.Naturforsch.,BChem.Sci., 54, 501, 1999.

GUHREG 2,6-Dimethylpyridinium hydrogen phthalate; C7 H10 N1 1+,C8 H5 O4 1-; Zhi Min Jin, Yuan Jiang Pan, Duan Jun Xu, Yuan Zhi Xu; J.Chem.Cryst., 30, 119, 2000.

GUKSOU 2-Aminopyridinium bis(benzenesulfonyl)amidate; C5 H7 N2 1+,C12 H10 N1 O4 S2 1-;

K.Wijaya, O.Moers, P.G.Jones, A.Blaschette; Acta Crystallogr.,Sect.C: Cryst.Struct.Commun., 57, 167, 2001.

HAXFER 3,5-Dimethylpyridinium 3,5-dinitrobenzoate 3,5-dinitrobenzoic acid; C7 H10 N1 1+,C7

H3 N2 O6 1-,C7 H4 N2 O6; L.B.Jerzykiewicz, T.Lis, Z.Malarski, E.Grech; J.Crystallogr.Spectrosc.Res., 23, 805, 1993.

HEYKAX bis(3-Methylpyridinium) dihydrogenpyromellitate; C10 H4 O8 2-,2(C6 H8 N1 1+); K.Biradha, M.J.Zaworotko; Crystal Engineering , 1, 67, 1998.

HEYQAD bis(4-Methylpyridinium) dihydrogenpyromellitate; C10 H4 O8 2-,2(C6 H8 N1 1+); K.Biradha, M.J.Zaworotko; Crystal Engineering , 1, 67, 1998.

HEYQEH bis(2-Ethylpyridinium) dihydrogenpyromellitate; C10 H4 O8 2-,2(C7 H10 N1 1+);

K.Biradha, M.J.Zaworotko; Crystal Engineering , 1, 67, 1998.

HEYQOR bis(2,4,6-Trimethylpyridinium) dihydrogenpyromellitate; C10 H4 O8 2-,2(C8 H12 N1 1+); K.Biradha, M.J.Zaworotko; Crystal Engineering , 1, 67, 1998.

HEYQOR01 bis(2,4,6-Trimethylpyridinium) dihydrogenpyromellitate; C10 H4 O8 2-,2(C8 H12 N1 1+); K.Biradha, M.J.Zaworotko; Crystal Engineering , 1, 67, 1998.

HIMGAL 1,3,5-Trihydroxybenzene tris(4-methylpyridine); C6 H6 O3,3(C6 H7 N1); K.Biradha, M.J.Zaworotko; J.Am.Chem.Soc., 120, 6431, 1998.

HIMGEP 1,3,5-Trihydroxybenzene tris(2,4-dimethylpyridine); C6 H6 O3,3(C7 H9 N1); K.Biradha, M.J.Zaworotko; J.Am.Chem.Soc., 120, 6431, 1998.

HIQVOS 2-Aminopyridinium di(methanesulfonyl)amidate $\quad 1,4,7,1 \mathrm{O}, 13,16$-hexa-oxa-cyclooctadecane; C12 H24 O6,C2 H6 N1 O4 S2 1-,C5 H7 N2 1+; D.Henschel, K.Wijaya, P.G.Jones, A.Blaschette; Acta Crystallogr.,Sect.C: Cryst.Struct.Commun., 55, 664, 1999.

HOPXUF 2,8,14,20-tetramethyl-4,6,10,12,16,18,22,24-octahydroxycalix[4]arene tetrakis(4picoline) nitromethane clathrate; C32 H32 O8,C1 H3 N1 O2,4(C6 H7 N1); L.R.MacGillivray, J.L.Atwood; Chem.Commun., , 181, 1999. 
HUVYIG (3R,6S,7R,8S,8aS)-Methyl 7,8-dihydroxy-5-oxo-6-(trifluoromethanesulfonyloxy)hexahydro-5H-(1,3)thiazolo(3,2-a)pyridine-3-carboxylate pyridine solvate; C10 H12 F3 N1 O8 S2,C5 H5 N1; P.Tremmel, J.Brand, V.Knapp, A.Geyer; Eur.J.Org.Chem., , 878, 2003.

HUYXII 1H-Nicotinamidium 3,5-dinitrosalicylate; C6 H7 N2 O1 1+,C7 H3 N2 O7 1-; M.Koman, L.Martiska, D.Valigura, T.Glowiak; Acta Crystallogr.,Sect.E: Struct.Rep.Online , 59, o441, 2003.

HUZHEP Ammonium 2-mercaptopyridine-3-carboxylate monohydrate; H4 N1 1+,C6 H4 N1 O2 S1 1-,H2 O1; G.Smith, D.S.Sagatys; Acta Crystallogr.,Sect.E: Struct.Rep.Online , 59, o540, 2003. IBITES 5-((1E)-2-(4-Hydroxyphenyl)ethenyl)-4,7-dimethoxy-3-methyl-2H-1-benzopyran-2-one pyridine solvate; C20 H18 O5,C5 H5 N1; D.Chavez, Hee-Byung Chai, T.E.Chagwedera, Qi Gao, N.R.Farnsworth, G.A.Cordell, J.M.Pezzuto, A.D.Kinghorn; Tetrahedron Lett., 42, 3685, 2001.

IDAHOK Pyridinium 4-nitrobenzoate 4-nitrobenzoic acid; C5 H6 N1 1+,C7 H4 N1 O4 1-,C7 H5 N1 O4; C.J.Carrow, K.A.Wheeler; Crystal Engineering , 1, 263, 1998.

IDAJAY 2-Vinylpyridinium 3,5-dinitrobenzoate 3,5-ninitrobenzoic acid; C7 H8 N1 1+,C7 H3 N2

O6 1-,C7 H4 N2 O6; C.J.Carrow, K.A.Wheeler; Crystal Engineering , 1, 263, 1998.

IDUNIE 3-Hydroxypyridinium hyrido-bis(4-nitrobenzoate); C5 H6 N1 O1 1+,C14 H9 N2 O8 1-; D.E.Lynch, J.Lad, G.Smith, S.Parsons; Crystal Engineering , 2, 65, 1999.

IDUNOK 3-Hydroxypyridinium 2,4-dinitrobenzoate; C5 H6 N1 O1 1+,C7 H3 N2 O6 1-; D.E.Lynch, J.Lad, G.Smith, S.Parsons; Crystal Engineering , 2, 65, 1999.

IHAQOX bis(2-Amino-3-methylpyridinium) o-phthalate; 2(C6 H9 N2 1+),C8 H4 O4 2-; Zhi Min Jin, Yuan Jiang Pan, Mao Lin Hu, Shen Liang; J.Chem.Cryst., 31, 191, 2001.

ILUCIB Pyridinium 3,3',4-tri-O-methylflavellagate; C5 H6 N1 1+,C17 H11 O9 1-; G.R.Pettit, Yanhui Meng, D.L.Herald, K.A.N.Graham, R.K.Pettit, D.L.Doubek; J.Nat.Prod., 66, 1065, 2003. IMIPEZ 2-Amino-5-methylpyridinium 3-(4-hydroxy-3-methoxyphenyl)-2-propenoate monohydrate; C6 H9 N2 1+,C10 H9 O4 1-,H2 O1; Richeng Xuan, Yuehua Wan, Weixiao Hu, Zhongyu Yang, Dongping Cheng, Rirong Xuan; Acta Crystallogr.,Sect.E: Struct.Rep.Online, 59, o1704, 2003.

IPOZAO Pyridinium-3-carboxamide picrate; C6 H7 N2 O1 1+,C6 H2 N3 O7 1-; Yue-Ping Fang, Ming-Hua Zeng, Rong-Ying Zeng, Hong Liang, Xiang-Hui Yi, Kai-Bei Yu; Hecheng Huaxue(Chin.)(Chin.J.Synth.Chem.), 11, 128, 2003.

ISNICA Isonicotinic acid; C6 H5 N1 O2; F.Takusagawa, A.Shimada; Acta Crystallogr.,Sect.B: Struct.Crystallogr.Cryst.Chem., 32, 1925, 1976.

ITUXOK N-(2-Phenyl-1H-indol-3-carbaldehyde)-N'-(4-nitrophenyl)hydrazone pyridine solvate; C21 H16 N4 O2,C5 H5 N1; L.N.Kuleshova, M.Yu.Antipin, V.N.Khrustalev, D.V.Gusev, 
G.V.Grintselev-Knyazev，E.S.Bobrikova; Kristallografiya(Russ.)(Crystallogr.Rep.) ，48，645, 2003.

IVOFEE 4,4'-bis(Diphenylhydroxymethyl)biphenyl bis(3-picoline) clathrate; C38 H30 O2,2(C6

H7 N1); L.R.Nassimbeni, Hong Su, E.Weber, K.Skobridis; Cryst.Growth Des., 4, 85, 2004.

IVOFOO 4,4'-bis(Diphenylhydroxymethyl)biphenyl bis(4-picoline) clathrate; C38 H30 O2,2(C6

H7 N1); L.R.Nassimbeni, Hong Su, E.Weber, K.Skobridis; Cryst.Growth Des., 4, 85, 2004.

IVOFOO01 4,4'-bis(Diphenylhydroxymethyl)biphenyl bis(4-picoline) clathrate; C38 H30 O2,2(C6

H7 N1); L.R.Nassimbeni, Hong Su, E.Weber, K.Skobridis; Cryst.Growth Des., 4, 85, 2004.

IXOHAE 4,4'-Diethyl-5,5'-dicarboxy-2,2'-bithiazole pyridine solvate; C12 H12 N2 O4 S2,2(C5 H5

N1); M.D.Curtis, J.Cao, J.W.Kampf; Private Communication , , , 2004.

IYUNOF Terephthalic acid pyridine solvate; C8 H6 O4,2(C5 H5 N1); S.H.Dale, M.R.J.Elsegood,

M.Hemmings, A.L.Wilkinson; CrystEngComm , 6, 207, 2004.

IYUPAT Pyridinium hydrogen phthalate; C5 H6 N1 1+,C8 H5 O4 1-; S.H.Dale, M.R.J.Elsegood,

M.Hemmings, A.L.Wilkinson; CrystEngComm , 6, 207, 2004.

IYUPEX Isophthalic acid pyridine solvate; C8 H6 O4,C5 H5 N1; S.H.Dale, M.R.J.Elsegood,

M.Hemmings, A.L.Wilkinson; CrystEngComm , 6, 207, 2004.

IYUPIB Pyridinium trihydrogen pyromellitate; C5 H6 N1 1+,C10 H5 O8 1-; S.H.Dale,

M.R.J.Elsegood, M.Hemmings, A.L.Wilkinson; CrystEngComm , 6, 207, 2004.

JOJJUN 3-Hydroxypyridine 2,4,6-trinitrobenzoic acid; C5 H6 N1 O1 1+,C7 H2 N3 O8 1-;

D.E.Lynch, G.Smith, K.A.Byriel, C.H.L.Kennard; Acta Crystallogr.,Sect.C:

Cryst.Struct.Commun., 48, 533, 1992.

KIXCUP 4-Hydroxypyridine-2,6-dicarboxylic acid monohydrate; C7 H5 N1 O5,H2 O1; A.K.Hall,

J.M.Harrowfield, B.W.Skelton, A.H.White; Acta Crystallogr.,Sect.C: Cryst.Struct.Commun., 56, 448, 2000.

KOBMAP 4-(Dimethylamino)pyridinium 2,4-dinitrobenzoate; C7 H11 N2 1+,C7 H3 N2 O6 1-;

H.Hosomi, S.Ohba, Y.Ito; Acta Crystallogr.,Sect.C: Cryst.Struct.Commun., 56, e149, 2000.

KOBNAQ 4-(Dimethylamino)pyridinium 3,4-dinitrobenzoate; C7 H11 N2 1+,C7 H3 N2 O6 1-;

H.Hosomi, S.Ohba, Y.Ito; Acta Crystallogr.,Sect.C: Cryst.Struct.Commun., 56, e149, 2000.

KOBNOE 4-(Dimethylamino)pyridinium 3,5-dinitrobenzoate; C7 H11 N2 1+,C7 H3 N2 O6 1-;

H.Hosomi, S.Ohba, Y.Ito; Acta Crystallogr.,Sect.C: Cryst.Struct.Commun., 56, e149, 2000.

KOCHOZ 2,6-Lutidine 1,1,2,2-tetraphenylethane-1,2-diol clathrate; C7 H9 N1,C26 H22 O2;

S.A.Bourne, L.R.Nassimbeni, F.Toda; J.Chem.Soc.,Perkin Trans.2 , , 1335, 1991.

KOXREU Pyridinium trihydrogen dimalonate; C3 H3 O4 1-,C3 H4 O4,C5 H6 N1 1+; K.Djinovic, L.Golic; Acta Crystallogr.,Sect.C: Cryst.Struct.Commun., 48, 1046, 1992. 
KUFBOC (2,4-Dichlorophenoxy)acetic acid 3-hydroxypyridine; C8 H5 Cl2 O3 1-,C5 H6 N1 O1

1+; K.A.Byriel, C.H.L.Kennard, D.E.Lynch, G.Smith, J.G.Thompson; Aust.J.Chem., 45, 969, 1992.

KUSZUT 4-Methylpyridinium trifluoroacetate; C6 H8 N1 1+,C2 F3 O2 1-; Z.Dega-Szafran, M.Gdaniec, M.Grundwald-Wyspianska, Z.Kosturkiewicz, J.Koput, P.Krzyzanowski, M.Szafran; J.Mol.Struct., 270, 99, 1992.

KUTBAC 4-Dimethylaminopyridinium trifluoroacetate; C7 H11 N2 1+,C2 F3 O2 1-; Z.DegaSzafran, M.Gdaniec, M.Grundwald-Wyspianska, Z.Kosturkiewicz, J.Koput, P.Krzyzanowski, M.Szafran; J.Mol.Struct., 270, 99, 1992.

LACVOB bis(4-(Dimethylamino)pyridinium) 5-phosphonobenzene-1,3-diphosphonate; 2(C7 H11 N2 1+),C6 H7 O9 P3 2-; M.Mehring; Eur.J.Inorg.Chem., , 3240, 2004.

LACVUH 4-(Dimethylamino)pyridinium 3,5-bis(phosphono)benzenephosphonate; C7 H11 N2 1+,C6 H8 O9 P3 1-; M.Mehring; Eur.J.Inorg.Chem., , 3240, 2004.

LEJROH Pyrazine-2,6-dicarboxylic acid 3-hydroxypyridine; C6 H4 N2 O4,C5 H5 N1 O1; D.E.Lynch, G.Smith, K.A.Byriel, C.H.L.Kennard, A.K.Whittaker; Aust.J.Chem., 47, 309, 1994.

LEWRUA 2-Aminopyridinium 2,6-dihydroxybenzoate; C5 H7 N2 1+,C7 H5 O4 1-; D.E.Lynch, G.Smith, D.Freney, K.A.Byriel, C.H.L.Kennard; Aust.J.Chem., 47, 1097, 1994.

LEZJIH Pyridinium 2,6-dihydroxybenzoate; C7 H5 O4 1-,C5 H6 N1 1+; M.Gdaniec, M.Gilski, G.S.Denisov; Acta Crystallogr.,Sect.C: Cryst.Struct.Commun., 50, 1622, 1994.

LILHIX 2-Aminopyridinium propynoate; C5 H7 N2 1+,C3 H1 O2 1 -; K.A.Wheeler, B.M.Foxman; Chem.Mater., 6, 1330, 1994.

LOLSUA 2-Methylpyridine pentachlorophenol; C6 H7 N1,C6 H1 Cl5 O1; T.Steiner, C.C.Wilson, I.Majerz; Chem.Commun., , 1231, 2000.

LONRAH 2-Aminopyridinium bis(methanesulfonyl)amide; C5 H7 N2 1+,C2 H6 N1 O4 S2 1-; O.Moers, K.Wijaya, I.Lange, A.Blaschette, P.G.Jones; Z.Naturforsch.,BChem.Sci., 55, 738, 2000.

LONROV 2-Amino-6-methylpyridinium bis(methanesulfonyl)amide hemihydrate; C6 H9 N2 1+,C2 H6 N1 O4 S2 1-,0.5(H2 O1); O.Moers, K.Wijaya, I.Lange, A.Blaschette, P.G.Jones; Z.Naturforsch.,BChem.Sci., 55, 738, 2000.

LOTBOL 4-Dimethylaminopyridinium 5-chloro-1,3-bis(benzyloxycarbonyl)-2-indole-2-oxide; C24 H17 Cl1 N1 O5 1-,C7 H1 1 N2 1+; M.Porcs-Makkay, G.Argay, A.Kalman, G.Simig; Tetrahedron , 56, 5893, 2000.

LUNMAI Isonicotinamide cinnamic acid; C6 H6 N2 O1,C9 H8 O2; C.B.Aakeroy, A.M.Beatty, B.A.Helfrich; J.Am.Chem.Soc., 124, 14425, 2002. 
LUNMEM Isonicotinamide 3-hydroxybenzoic acid; C6 H6 N2 O1,C7 H6 O3; C.B.Aakeroy, A.M.Beatty, B.A.Helfrich; J.Am.Chem.Soc., 124, 14425, 2002.

LUNMUC Isonicotinamide 12-bromododecanoic acid; C6 H6 N2 O1,C12 H23 Br1 O2; C.B.Aakeroy, A.M.Beatty, B.A.Helfrich; J.Am.Chem.Soc., 124, 14425, 2002.

LUNNAJ Isonicotinamide monochloroacetic acid; C6 H6 N2 O1,C2 H3 C11 O2; C.B.Aakeroy, A.M.Beatty, B.A.Helfrich; J.Am.Chem.Soc., 124, 14425, 2002.

LUNNEN Isonicotinamide fumaric acid monoethyl ester; C6 H6 N2 O1,C6 H8 O4; C.B.Aakeroy, A.M.Beatty, B.A.Helfrich; J.Am.Chem.Soc., 124, 14425, 2002.

LUNNIR bis(Isonicotinamide) 4-ketopimelic acid; 2(C6 H6 N2 O1),C7 H10 O5; C.B.Aakeroy, A.M.Beatty, B.A.Helfrich; J.Am.Chem.Soc., 124, 14425, 2002.

LUNNOX bis(Isonicotinamide) fumaric acid; $2(\mathrm{C} 6 \mathrm{H} 6 \mathrm{~N} 2$ O1),C4 H4 O4; C.B.Aakeroy, A.M.Beatty, B.A.Helfrich; J.Am.Chem.Soc., 124, 14425, 2002.

LUNNUD bis(Isonicotinamide) succinic acid; 2(C6 H6 N2 O1),C4 H6 O4; C.B.Aakeroy, A.M.Beatty, B.A.Helfrich; J.Am.Chem.Soc., 124, 14425, 2002.

LUNNUD01 Succinic acid bis(isonicotinamide); C4 H6 O4,2(C6 H6 N2 O1); P.Vishweshwar, A.Nangia, V.M.Lynch; Cryst.Growth Des., 3, 783, 2003.

LUNPAL Isonicotinamide d,l-mandelic acid; C6 H6 N2 O1,C8 H8 O3; C.B.Aakeroy, A.M.Beatty, B.A.Helfrich; J.Am.Chem.Soc., 124, 14425, 2002.

LUNPEP bis(Isonicotinamide) thiodiglycolic acid; 2(C6 H6 N2 O1),C4 H6 O4 S1; C.B.Aakeroy, A.M.Beatty, B.A.Helfrich; J.Am.Chem.Soc., 124, 14425, 2002.

LUPZOL 2-Aminopyridinium benzoate; C5 H7 N2 1+,C7 H5 O2 1-; M.Odabasoglu, O.Buyukgungor, P.Lonnecke; Acta Crystallogr.,Sect.C: Cryst.Struct.Commun., 59, o51, 2003.

MAHWOI 3,5-Lutidinium 2-chloro-4-nitrobenzoate 2-chloro-4-nitrobenzoic acid; C7 H10 N1 1+,C7

H3 Cl1 N1 O4 1-,C7 H4 Cl1 N1 O4; H.Ishida, B.Rahman, S.Kashino; Acta Crystallogr.,Sect.E: Struct.Rep.Online, 60, o1661, 2004.

MAHWUO 3-Cyanopyridine 2-chloro-4-nitrobenzoic acid; C6 H4 N2,C7 H4 Cl1 N1 O4; H.Ishida,

T.Fukunaga; Acta Crystallogr.,Sect.E: Struct.Rep.Online , 60, o1664, 2004.

MAJTOH 3-bromopyridinium tribromoacetate; C5 H5 Br1 N1 1+,C2 Br3 O2 1-; P.G.Jones,

V.Lozano; Acta Crystallogr.,Sect.C: Cryst.Struct.Commun., 60, o876, 2004.

MIBYEB 2,6-Dimethylpyridinium hydrogen fumarate; C7 H10 N1 1+,C4 H3 O4 1-; Yuan Jiang Pan, Zhi Min Jin, Cui Rong Sun, Chong Wen Jiang; Chem.Lett., , 1008, 2001.

MIZMUD 4-Dimethylaminopyridinium 5-chloro-1-ethoxycarbonyl-3-(1-oxy-1-(2thienyl)methylene)-2-oxo-2,3-dihydroindole; C7 H11 N2 1+,C16 H11 C11 N1 O4 S1 1-; M.Porcs-Makkay, G.Simig; Organic Process Research \& Development , 4, 10, 2000. 
MOPYAR Cyanuric acid pyridine; C3 H3 N3 O3,C5 H5 N1; K.Sivashankar; Proc.Indian Acad.Sci.,Chem.Sci., 112, 607, 2000.

MPYDCX01 3-Thioxo-2-pyridinecarboxylic acid; C6 H5 N1 O2 S1; P.E.Bourne, M.R.Taylor; Acta Crystallogr.,Sect.C: Cryst.Struct.Commun., 39, 266, 1983.

NAMTAW Pyridinium phenylphosphonite hexafluoro-2,2-propanediol solvate; 2(C5 H6 N1 1+),2(C6 H6 O2 P1 1-),3(C3 H2 F6 O2); J.R.Goerlich, A.Fischer, P.G.Jones, R.Schmutzler; J.Fluorine Chem., 72, 69, 1995.

NEYLOS01 2,8,14,20-Tetramethyl-4,6,10,12,16,18,22,24-octahydroxy-calix(4)arene pyridine clathrate; C32 H32 O8,6(C5 H5 N1); W.Iwanek, R.Frohlich, M.Urbaniak, C.Nather, J.Mattay; Tetrahedron , 54, 14031, 1998.

NICOAC02 Nicotinic acid; C6 H5 N1 O2; A.Kutoglu, C.Scheringer; Acta Crystallogr.,Sect.C: Cryst.Struct.Commun., 39, 232, 1983.

NICOAM01 Nicotinamide; C6 H6 N2 O1; Y.Miwa, T.Mizuno, K.Tsuchida, T.Taga, Y.Iwata; Acta Crystallogr.,Sect.B: Struct.Sci., 55, 78, 1999.

NONMIM 1,1-bis(4-Hydroxyphenyl)cyclohexane 3-picoline clathrate; C18 H20 O2,C6 H7 N1; M.R.Caira, A.Horne, L.R.Nassimbeni, F.Toda; J.Mater.Chem., 7, 2145, 1997.

NONMOS 1,1-bis(4-Hydroxyphenyl)cyclohexane 4-picoline clathrate; C18 H20 O2,C6 H7 N1; M.R.Caira, A.Horne, L.R.Nassimbeni, F.Toda; J.Mater.Chem., 7, 2145, 1997.

NUKBIE 4-(Dimethylamino)pyridinium 2,6-dichloro-4-nitrophenolate; C7 H11 N2 1+,C6 H2 Cl2 N1 O3 1-; I.Majerz, W.Sawka-Dobrowolska, L.Sobczyk; J.Mol.Struct., 375, 37, 1996.

NUNLUD Methylphenylammonium hydrogen 2,6-pyridinecarboxylate; C7 H10 N1 1+,C7 H4 N1 O4 1-; Seik Weng Ng, M.M.Turnbull; Acta Crystallogr.,Sect.C: Cryst.Struct.Commun., 54, 1025, 1998.

OBELIQ 1,4-bis(9-Hydroxy-9-fluorenyl)benzene bis(3,5-lutidine) clathrate; C32 H22 O2,2(C7 H9 N1); M.R.Caira, L.R.Nassimbeni, D.Vujovic, E.Weber, A.Wierig; Struct.Chem., 10, 205, 1999. OBELOW 1,4-bis(9-Hydroxy-9-fluorenyl)benzene bis(2,6-lutidine) clathrate; C32 H22 O2,2(C7 H9 N1); M.R.Caira, L.R.Nassimbeni, D.Vujovic, E.Weber, A.Wierig; Struct.Chem., 10, 205, 1999. OCAKAF 3-Hydroxypyridinium 3-(carboxymethoxy)phenoxyacetate; C5 H6 N1 O1 1+,C10 H9 O6 1-; Shan Gao, Ji-Wei Liu, Li-Hua Huo, Hui Zhao, S.W.Ng; Acta Crystallogr.,Sect.E: Struct.Rep.Online , 60, o1854, 2004.

OCANIQ bis(3-Picolinium) chloranilate dihydrate; 2(C6 H8 N1 1+),C6 Cl2 O4 2-,2(H2 O1); H.Ishida; Acta Crystallogr.,Sect.E: Struct.Rep.Online , 60, o1900, 2004. 
OFUZUK Decane-1,10-diammonium bis(3-carboxy-pyridine-5-carboxylate) monohydrate; C10 H26 N2 2+,2(C7 H4 N1 O4 1-),H2 O1; A.M.Beatty, C.M.Schneider, A.E.Simpson, J.L.Zaher; CrystEngComm , 4, 282, 2002.

OGABAZ Dodecane-1,12-diammonium bis(3-carboxy-pyridine-5-carboxylate) monohydrate; C12 H30 N2 2+,2(C7 H4 N1 O4 1-),H2 O1; A.M.Beatty, C.M.Schneider, A.E.Simpson, J.L.Zaher; CrystEngComm , 4, 282, 2002.

OMIHIB Mellitic acid tetrakis(4-aminopyridine) dihydrate; C12 H2 O12 4-,4(C5 H7 N2 1+),2(H2 O1); I.Karle, R.D.Gilardi, C.C.Rao, K.M.Muraleedharan, S.Ranganathan; J.Chem.Cryst., 33, 727, 2003.

PABBAV 4-Acetylpyridine pentachlorophenol; C7 H7 N1 O1,C6 H1 C15 O1; I.Majerz, Z.Malarski, W.Sawka-Dobrowolska; J.Mol.Struct., 249, 109, 1991.

PAHZAA 3-Hydroxypyridinium hydrogen tartronate; C5 H6 N1 O1 1+,C3 H3 O5 1-; T.Fukunaga, S.Kashino, H.Ishida; Acta Crystallogr.,Sect.C: Cryst.Struct.Commun., 60, o718, 2004.

PARNAX beta-2,4,6-Trimethylpyridinium pentachlorophenolate; C8 H12 N1 1+,C6 Cl5 O1 1-; I.Majerz, Z.Malarski, W.Sawka-Dobrowolska; J.Mol.Struct., 273, 161, 1992.

PEBFIL 2-Amino-5-nitropyridinium L-monohydrogentartrate; C5 H6 N3 O2 1+,C4 H5 O6 1-; J.Zyss, R.Masse, M.Bagieu-Beucher, J.P.Levy; Adv.Mater., 5, 120, 1993.

PEBFIL01 2-Amino-5-nitropyridinium L-(+)-tartrate; C5 H6 N3 O2 1+,C4 H5 O6 1-; O.Watanabe, T.Noritake, Y.Hirose, A.Okada, T.Kurauchi; J.Mater.Chem., 3, 1053, 1993.

PEQBES Octadecanoic acid 3-pyridinecarboxamide; C18 H36 O2,C6 H6 N2 O1; M.Amai, T.Endo, H.Nagase, H.Ueda, M.Nakagaki; Acta Crystallogr.,Sect.C: Cryst.Struct.Commun., 54, 1367, 1998.

PEZXIB 3-Methylpyridinium 2,6-dichloro-4-nitrophenoxide; C6 H2 Cl2 N1 O3 1-,C6 H8 N1 1+; I.Majerz, W.Sawka-Dobrowolska, L.Sobczyk; J.Mol.Struct., 297, 177, 1993.

PIJVEJ bis(1,2,3,4,5-Pentamethyl-2,4-cyclopentadien-1-yl)methanol pyridine solvate; C21 H32

O1,C5 H5 N1; P.Jutzi, A.Mix, T.Lindermeier, H.-G.Stammler, B.Neumann; Chem.Ber., 127, 107, 1994.

POCVUY 3,4-Dimethylpyridinium 2,6-dichloro-4-nitrophenolate; C7 H10 N1 1+,C6 H2 Cl2 N1 O3 1-; I.Majerz, W.Sawka-Dobrowolska, L.Sobczyk; J.Mol.Struct., 319, 1, 1994.

PUHRIT 3,5-Dimethylpyridinium 3,5-dinitrobenzoate; C7 H10 N1 1+,C7 H3 N2 O6 1-;

L.B.Jerzykiewicz, Z.Malarski, L.Sobczyk, T.Lis, E.Grech; J.Mol.Struct., 440, 175, 1998.

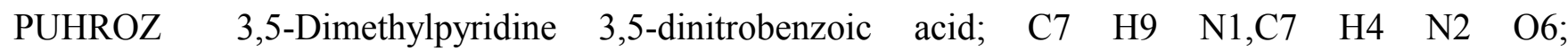

L.B.Jerzykiewicz, Z.Malarski, L.Sobczyk, T.Lis, E.Grech; J.Mol.Struct., 440, 175, 1998. 
PUJDUT Ammonium pentafluorothiophenolate pyridine solvate; C6 F5 S1 1-,H4 N1 1+,C5 H5

N1; S.Chadwick, U.Englich, B.Noll, K.Ruhlandt-Senge; Inorg.Chem., 37, 4718, 1998.

PUMQAP01 4-Dimethylaminopyridinium 4-nitrophenolate 4-nitrophenol; C7 H11 N2 1+,C6 H4 N1

O3 1-,C6 H5 N1 O3; N.Vembu, M.Nallu, E.C.Spencer, J.A.K.Howard; Acta Crystallogr.,Sect.E: Struct.Rep.Online, 59, o1192, 2003.

PYRPIC02 Pyridinium picrate; C5 H6 N1 1+,C6 H2 N3 O7 1-; M.Botoshansky, F.H.Herbstein, M.Kapon; Acta Crystallogr.,Sect.B: Struct.Sci., 50, 191, 1994.

QAFFIM Pyridine formic acid; C5 H5 N1,C1 H2 O2; D.Wiechert, D.Mootz; Angew.Chem.,Int.Ed., 38, 1974, 1999.

QAFFOS Pyridinium formate tris(formic acid); C5 H6 N1 1+,C1 H1 O2 1-,3(C1 H2 O2);

D.Wiechert, D.Mootz; Angew.Chem.,Int.Ed., 38, 1974, 1999.

QAMSUS 3-Carboxypyridinium chloride; C6 H6 N1 O2 $\quad$ H+,Cl1 1-; M.Slouf; Acta Crystallogr.,Sect.E: Struct.Rep.Online , 57, o61, 2001.

QECROF N,N'-Ditosyl-p-phenylenediamine bis(pyridine) clathrate; C20 H20 N2 O4 S2,2(C5 H5 N1); H.Bock, N.Nagel, C.Nather; Z.Naturforsch.,BChem.Sci., 53, 1425, 1998.

QIGVUX 1,3-dimethylbarbituric acid 2,6-diaminopyridine; C5 H8 N3 1+,C6 H7 N2 O3 1-; V.Bertolasi, P.Gilli, V.Ferretti, G.Gilli; New J.Chem.(Nouv.J.Chim.) , 25, 408, 2001.

QIGWAE 5'-hydroxy-5,5'-bi(1,3-dimethylbarbituric acid) 4-aminopyridine; C12 H13 N4 O7 1-,C5

H7 N2 1+; V.Bertolasi, P.Gilli, V.Ferretti, G.Gilli; New J.Chem.(Nouv.J.Chim.) , 25, 408, 2001.

QIJBEQ bis(2,2'-Ethylenedioxydibenzaldehyde bis(thiocarbohydrazide)) pyridine solvate; C34

H32 N8 O4 S2,3(C5 H5 N1); S.Chantrapromma, I.A.Razak, H.-K.Fun, C.Karalai, Hao Zhang,

Fu-Xin Xie, Yu-Peng Tian, Wen Ma, Yin-Han Zhang, Shi-Sheng Ni; Acta Crystallogr.,Sect.C:

Cryst.Struct.Commun., 57, 289, 2001.

QOQVOH Pyridinium methylsulfonate; C5 H6 N1 1+,C1 H3 O3 S1 1-; M.Bolte, C.Griesinger, P.Sakhaii; Acta Crystallogr.,Sect.E: Struct.Rep.Online , 57, o458, 2001.

QOYKOE 2,2'-Dihydroxy-1,1'-binaphthyl bis(2-picoline) clathrate; C20 H14 O2,2(C6 H7 N1);

L.R.Nassimbeni, Hong Su; Acta Crystallogr.,Sect.B: Struct.Sci., 57, 394, 2001.

QOYKUK 2,2'-Dihydroxy-1,1'-binaphthyl bis(3-picoline) clathrate; C20 H14 O2,2(C6 H7 N1);

L.R.Nassimbeni, Hong Su; Acta Crystallogr.,Sect.B: Struct.Sci., 57, 394, 2001.

QOYLAR 2,2'-Dihydroxy-1,1'-binaphthyl bis(4-picoline) clathrate; C20 H14 O2,2(C6 H7 N1);

L.R.Nassimbeni, Hong Su; Acta Crystallogr.,Sect.B: Struct.Sci., 57, 394, 2001.

QUICNA01 Quinolinic acid; C7 H5 N1 O4; A.Kvick, T.F.Koetzle, R.Thomas, F.Takusagawa; J.Chem.Phys., 60, 3866, 1974. 
QUICNA02 Quinolinic acid; C7 H5 N1 O4; F.Takusagawa, T.F.Koetzle; Acta Crystallogr.,Sect.B:

Struct.Crystallogr.Cryst.Chem., 34, 1149, 1978.

QUICNA10 Quinolinic acid; C7 H5 N1 O4; F.Takusagawa, K.Hirotsu, A.Shimada; Bull.Chem.Soc.Jpn., 46, 2372, 1973.

QUSLEV Pyridinium bis(methylsulfonyl)amide; C5 H6 N1 1+,C2 H6 N1 O4 S2 1-; O.Moers, I.Lange, K.Wijaya, A.Blaschette, P.G.Jones; Z.Naturforsch.,BChem.Sci., 56, 1041, 2001.

QUSLOF 2,6-Dimethylpyridinium bis(methylsulfonyl)amide; C7 H10 N1 1+,C2 H6 N1 O4 S2 1-; O.Moers, K.Wijaya, T.Hamann, A.Blaschette, P.G.Jones; Z.Naturforsch.,BChem.Sci., 56, 1052, 2001.

RACBED 3-Hydroxypyridinium 2,5-dihydroxybenzoate; C5 H6 $\mathrm{N} 1 \quad \mathrm{O} 1 \quad$ 1+,C7 $\mathrm{H} 5$ O4 1-; T.Fukunaga, S.Kashino, H.Ishida; Acta Crystallogr.,Sect.E: Struct.Rep.Online, 59, o420, 2003.

RAKQAV 4-Methylpyridine pentachlorophenol; C6 Cl5 O1 1-,C6 H8 N1 1+; T.Steiner, I.Majerz, C.C.Wilson; Angew.Chem.,Int.Ed., 40, 2651, 2001.

RAKQAV01 4-Methylpyridine pentachlorophenol; C6 Cl5 O1 1-,C6 H8 N1 1+; T.Steiner, I.Majerz, C.C.Wilson; Angew.Chem.,Int.Ed., 40, 2651, 2001.

RAKQAV02 4-Methylpyridine pentachlorophenol; C6 Cl5 O1 1-,C6 H8 N1 1+; T.Steiner, I.Majerz, C.C.Wilson; Angew.Chem.,Int.Ed., 40, 2651, 2001.

RALDUD 3-Aminopyridinium hydrogen squarate; C5 H7 N2 1+,C4 H1 O4 1-; V.Bertolasi, P.Gilli, V.Ferretti, G.Gilli; Acta Crystallogr.,Sect.B: Struct.Sci., 57, 591, 2001.

RALFAL 4-Aminopyridinium hydrogen squarate; C5 H7 N2 1+,C4 H1 O4 1-; V.Bertolasi, P.Gilli, V.Ferretti, G.Gilli; Acta Crystallogr.,Sect.B: Struct.Sci., 57, 591, 2001.

RALQAW01 Calix(8)arene bis(pyridine) clathrate pyridine solvate; C56 H48 O8,6(C5 H5 N1); V.Bohmer, V.Brusko, K.Rissanen; Synthesis , , 1898, 2002.

RAPHEV01 Benzene-1,3,5-tricarboxylic acid tris(pyridine); C9 H6 O6,3(C5 H5 N1); S.H.Dale, M.R.J.Elsegood, M.Hemmings, A.L.Wilkinson; CrystEngComm , 6, 207, 2004.

RENGAS bis(Dicyclohexylammonium) 2,6-pyridinedicarboxylate dihydrate; C7 H3 N1 O4 2,2(C12 H24 N1 1+),2(H2 O1); Seik Weng Ng; Acta Crystallogr.,Sect.C: Cryst.Struct.Commun., 53, 986, 1997.

REPMUU 2,4,6-Trimethylpyridinium 2,6-dichloro-4-nitrophenolate; C8 H12 N1 1+,C6 H2 Cl2 N1 O3 1-; I.Majerz, W.Sawka-Dobrowolska, L.Sobczyk; Acta Phys.Pol.,A, 88, 349, 1995.

RIWJIQ 2-Amino-5-nitro-pyridine 3-nitro-2-pyridone; C5 H5 N3 O2,C5 H4 N2 O3; V.Velikova, K.Kossev, O.Angelova; Acta Crystallogr.,Sect.C: Cryst.Struct.Commun., 53, 1270, 1997.

ROGXIU 2-Aminonicotinic acid; C6 H6 N2 O2; A.J.Dobson, R.E.Gerkin; Acta Crystallogr.,Sect.C: Cryst.Struct.Commun., 53, 1427, 1997. 
RUJCII N-t-Butyloxycarbonyl-L-phenylalanine pyridine; C14 H19 N1 O4,C5 H5 N1;

L.V.Meervelt, C.Bruyneel, H.Morisse, T.Zeegers-Huyskens; J.Phys.Org.Chem., 10, 825, 1997.

RUVYIQ Pyridinium trifluoroacetate; C2 F3 O2 1-,C5 H6 N1 1+; G.T.R.Palmore, M.T.McBride-

Wieser; Acta Crystallogr.,Sect.C: Cryst.Struct.Commun., 53, 1904, 1997.

SAZGIJ 2,4-Dimethylpyridinium pentachlorophenolate; C7 H10 N1 1+,C6 Cl5 O1 1-; I.Majerz, Z.Malarski, T.Lis; J.Crystallogr.Spectrosc.Res., 19, 349, 1989.

SEGRUR 2-Amino-4-nitropyridine; C5 H5 N3 O2; J.Oszust, Z.Talik, A.Pietraszko, M.K.Marchewka, J.Baran; J.Mol.Struct., 415, 53, 1997.

SEJSUV 4-Picolinium hydrogen malonate; C6 H8 N1 1+,C3 H3 O4 1-; K.Djinovic, L.Golic, I.Leban; Acta Crystallogr.,Sect.C: Cryst.Struct.Commun., 46, 281, 1990.

SIRPOY trans-9,10-Dihydroxy-9,10-diphenyl-9,10-dihydroanthracene bis(4-vinylpyridine)

clathrate; C26 H20 O2,2(C7 H7 N1); D.R.Bond, M.R.Caira, G.A.Harvey, L.R.Nassimbeni, F.Toda; Acta Crystallogr.,Sect.B: Struct.Sci., 46, 771, 1990.

SIRPUE trans-9,10-Dihydroxy-9,10-diphenyl-9,10-dihydroanthracene bis(4-methylpyridine) clathrate; C26 H20 O2,2(C6 H7 N1); D.R.Bond, M.R.Caira, G.A.Harvey, L.R.Nassimbeni, F.Toda; Acta Crystallogr.,Sect.B: Struct.Sci., 46, 771, 1990.

TABMAK bis(Pyridinium) 4,4'-methylene-bis(3-hydroxy-2-naphthalenecarboxylate); 2(C5 H6 N1 1+),C23 H14 O6 2-; A.C.Blackburn, A.J.Dobson, R.E.Gerkin; Acta Crystallogr.,Sect.C: Cryst.Struct.Commun., 52, 1269, 1996.

TETXUL 2-Amino-5-nitropyridine chloroacetic acid; C5 H5 N3 O2,C2 H3 Cl1 O2; Y.Le Fur, M.Bagieu-Beucher, R.Masse, J.-F.Nicoud, J.-P.Levy; Chem.Mater., 8, 68, 1996.

TIBMUM 3,5-Dinitro-2,4,6-triaminopyridine; C5 H6 N6 O4; R.A.Hollins, L.H.Merwin,

R.A.Nissan, W.S.Wilson, R.Gilardi; J.Heterocycl.Chem., 33, 895, 1996.

TIWXIG 2-Aminopyridinium hydrogen squarate; C5 H7 N2 1+,C4 H1 O4 1-; I.L.Karle, D.Ranganathan, V.Haridas; J.Am.Chem.Soc., 118, 7128, 1996.

TOYLOI 2-Amino-5-nitropyridine 2-amino-nitropyridinium oxamate; C5 H5 N3 O2,C5 H6 N3 O2 1+,C2 H2 N1 O3 1-; C.B.Aakeroy, D.P.Hughes, M.Nieuwenhuyzen; J.Am.Chem.Soc., 118, 10134, 1996.

UHAPUO 2-Chloro-4-nitrobenzoic acid 4-benzoylpyridine; C7 H4 Cl1 N1 O4,C12 H9 N1 O1; T.Sugiyama, Jiben Meng, T.Matsuura; J.Mol.Struct., 611, 53, 2002.

UHAQAV 2-Chloro-5-nitrobenzoic acid 4-benzoylpyridine; C7 H4 Cl1 N1 O4,C12 H9 N1 O1; T.Sugiyama, Jiben Meng, T.Matsuura; J.Mol.Struct., 611, 53, 2002.

UHAQEZ 4-Chloro-3-nitrobenzoic acid 4-benzoylpyridine; C7 H4 Cl1 N1 O4,C12 H9 N1 O1; T.Sugiyama, Jiben Meng, T.Matsuura; J.Mol.Struct., 611, 53, 2002. 
UHAQID 5-Chloro-2-nitrobenzoic acid 4-benzoylpyridine; C7 H4 Cl1 N1 O4,C12 H9 N1 O1;

T.Sugiyama, Jiben Meng, T.Matsuura; J.Mol.Struct., 611, 53, 2002.

UHAQOJ 4-Chloro-2-nitrobenzoic acid 4-benzoylpyridine; C7 H4 Cl1 N1 O4,C12 H9 N1 O1;

T.Sugiyama, Jiben Meng, T.Matsuura; J.Mol.Struct., 611, 53, 2002.

UHAQUP 3-Chloro-2-nitrobenzoic acid 4-benzoylpyridine; C7 H4 Cl1 N1 O4,C12 H9 N1 O1;

T.Sugiyama, Jiben Meng, T.Matsuura; J.Mol.Struct., 611, 53, 2002.

UHEMOJ 3-(3-(3-(3-Hydroxy-3,3-diphenylpropynyl)phenylazo)phenyl)-1,1-diphenylprop-2-ynol

dipyridine clathrate; C42 H30 N2 O2,2(C5 H5 N1); J.L.Scott, A.P.Downie, M.Asami, K.Tanaka; CrystEngComm , 4, 580, 2002.

ULAWAF Oxalic acid bis(isonicotinamide); C2 H2 O4,2(C6 H6 N2 O1); P.Vishweshwar, A.Nangia, V.M.Lynch; Cryst.Growth Des., 3, 783, 2003.

ULAWEJ Malonic acid bis(isonicotinamide); C3 H4 O4,2(C6 H6 N2 O1); P.Vishweshwar, A.Nangia, V.M.Lynch; Cryst.Growth Des., 3, 783, 2003.

ULAWOT Glutaric acid bis(isonicotinamide); C5 H8 O4,2(C6 H6 N2 O1); P.Vishweshwar, A.Nangia, V.M.Lynch; Cryst.Growth Des., 3, 783, 2003.

ULAWUZ Adipic acid bis(isonicotinamide); C6 H10 O4,2(C6 H6 N2 O1); P.Vishweshwar, A.Nangia, V.M.Lynch; Cryst.Growth Des., 3, 783, 2003.

ULAXAG Glutaric acid isonicotinamide; C5 H8 O4,C6 H6 N2 O1; P.Vishweshwar, A.Nangia, V.M.Lynch; Cryst.Growth Des., 3, 783, 2003.

ULAXEK Adipic acid isonicotinamide; C6 H10 O4,C6 H6 N2 O1; P.Vishweshwar, A.Nangia, V.M.Lynch; Cryst.Growth Des., 3, 783, 2003.

ULUVUS 5-(Ethoxycarbonyl)pyridine-2-carboxylic acid; C9 H9 N1 O4; J.Gatjens, B.Meier, T.Kiss, E.M.Nagy, P.Buglyo, H.Sakurai, K.Kawabe, D.Rehder; Chem.-Eur.J., 9, 4924, 2003.

ULUWAZ 5-(Methoxycarbonyl)pyridine-2-carboxylic acid monohydrate; C8 H7 N1 O4,H2 O1; J.Gatjens, B.Meier, T.Kiss, E.M.Nagy, P.Buglyo, H.Sakurai, K.Kawabe, D.Rehder; Chem.Eur.J., 9, 4924, 2003.

ULUWED 5-(Isopropoxycarbonyl)pyridine-2-carboxylic acid; C10 H11 N1 O4; J.Gatjens, B.Meier, T.Kiss, E.M.Nagy, P.Buglyo, H.Sakurai, K.Kawabe, D.Rehder; Chem.-Eur.J., 9, 4924, 2003.

UNECEV 1,2,4,5-Benzenetetracarboxylic acid benzene-2,5-dicarboxylate-1,4-dicarboxylic acid bis(4-(dimethylamino)pyridinium) clathrate; $2(\mathrm{C} 7$ H11 N2 1+),C10 H6 O8,C10 H4 O8 2-; K.K.Arora, V.R.Pedireddi; J.Org.Chem., 68, 9177, 2003.

VAKTOR 4-Hydroxybenzoic acid isonicotinamide; C7 H6 O3,C6 H6 N2 O1; P.Vishweshwar, A.Nangia, V.M.Lynch; CrystEngComm , 5, 164, 2003. 
VAKTUX Resorcinol bis(isonicotinamide); C6 H6 O2,2(C6 H6 N2 O1); P.Vishweshwar, A.Nangia, V.M.Lynch; CrystEngComm , 5, 164, 2003.

VAKVEJ Phloroglucinol bis(isonicotinamide) dihydrate; C6 H6 O3,2(C6 H6 N2 O1),2(H2 O1); P.Vishweshwar, A.Nangia, V.M.Lynch; CrystEngComm , 5, 164, 2003.

VAKVIN Hydroquinone bis(isonicotinamide); C6 H6 O2,2(C6 H6 N2 O1); P.Vishweshwar, A.Nangia, V.M.Lynch; CrystEngComm , 5, 164, 2003.

VASYEU ellagic acid tetrakis(pyridine); C14 H6 O8,4(C5 H5 N1); M.Hasegawa, M.Terauchi, Y.Kikuchi, A.Nakao, J.Okubo, T.Yoshinaga, H.Hiratsuka, M.Kobayashi, T.Hoshi; Monatsh.Chem., 134, 811, 2003.

WADPEX Oxalic acid oxalate pyridinium clathrate dihydrate; C2 H2 O4,C2 H1 O4 1-,C5 H6 N1 1+,2(H2 O1); K.Rajagopal, R.V.Krishnakumar, S.Natarajan; Acta Crystallogr.,Sect.E: Struct.Rep.Online, 59, o742, 2003.

WANTUA 2-Amino-5-nitropyridinium monohydrogensulfate; C5 H6 N3 O2 1+,H1 O4 S1 1-; J.Pecaut, Y.le Fur, R.Masse; Acta Crystallogr.,Sect.B: Struct.Sci., 49, 535, 1993.

WISNOB bis(1,1,3,3,5,5-Hexaphenyltrisiloxane-1,5-diol) tris(pyridine); 2(C36 H32 O4 Si3),3(C5 H5 N1); B.O'Leary, T.R.Spalding, G.Ferguson, C.Glidewell; Acta Crystallogr.,Sect.B: Struct.Sci., 56, 273, 2000.

WOSBEL 2-Amino-6-methylpyridinium neobietate; C20 H29 O2 1-,C6 H9 N2 1+; Zhimin Jin, Yuanjiang Pan, Jiageng Liu, Duanjun Xu; J.Chem.Cryst., 30, 195, 2000.

WUJRIC 2,6-Diaminopyridinium 1,3-cyclopentanedionate monohydrate; C5 H8 N3 1+,C5 H5 O2 1-,H2 O1; V.Bertolasi, L.Pretto, P.Gilli, V.Ferretti, G.Gilli; New J.Chem.(Nouv.J.Chim.) , 26, $1559,2002$.

WUJSAV 2,6-Diaminopyridinium 5-methyl-1,3-cyclohexanedionate 5-methyl-1,3-

cyclohexanedione enol; C5 H8 N3 1+,C7 H9 O2 1-,C7 H10 O2; V.Bertolasi, L.Pretto, P.Gilli, V.Ferretti, G.Gilli; New J.Chem.(Nouv.J.Chim.), 26, 1559, 2002.

WUJSEZ 2,6-Diaminopyridinium tropolonate monohydrate; C5 H8 N3 1+,C7 H5 O2 1-,H2 O1; V.Bertolasi, L.Pretto, P.Gilli, V.Ferretti, G.Gilli; New J.Chem.(Nouv.J.Chim.) , 26, 1559, 2002.

WUQMIE 6-Methylnicotinamide; C7 H8 N2 O1; J.C.Schlenker, R.J.Staples; Z.Kristallogr.-New Cryst.Struct., 217, 555, 2002.

WUXDEY bis(2-Aminopyridinium) maleate; 2(C5 H7 N2 1+),C4 H2 O4 2-; O.Buyukgungor, M.Odabasoglu; Acta Crystallogr.,Sect.C: Cryst.Struct.Commun., 59, o105, 2003.

WUXYUJ 2,4-Dimethylpyridinium 2,5-dichloro-6-hydroxy-1,4-benzoquinone-3-olate; C6 H1 Cl2 O4 1-,C7 H10 N1 1+; H.Ishida, S.Kashino; Z.Naturforsch.,APhys.Sci., 57, 829, 2002. 
WUXZAQ 2,5-Dimethylpyridinium 2,5-dichloro-6-hydroxy-1,4-benzoquinone-3-olate; C6 H1 Cl2

O4 1-,C7 H10 N1 1+; H.Ishida, S.Kashino; Z.Naturforsch.,APhys.Sci., 57, 829, 2002.

WUXZEU 2,6-Dimethylpyridinium 2,5-dichloro-6-hydroxy-1,4-benzoquinone-3-olate; C6 H1 Cl2

O4 1-,C7 H10 N1 1+; H.Ishida, S.Kashino; Z.Naturforsch.,APhys.Sci., 57, 829, 2002.

WUXZIY 3,4-Dimethylpyridinium 2,5-dichloro-6-hydroxy-1,4-benzoquinone-3-olate; C6 H1 Cl2

O4 1-,C7 H10 N1 1+; H.Ishida, S.Kashino; Z.Naturforsch.,APhys.Sci., 57, 829, 2002.

XAJVIO 2-Hydroxy-6-methylpyridinium chloride monohydrate; C6 H8 N1 O1 1+,Cl1 1-,H2 O1;

S.Parsons, A.Graham, R.Winpenny, P.Wood; Private Communication , , , 2004.

XEGNUS cis-cis-1,3,5-Trimethylcyclohexane-1,3,5-tricarboxylic acid 2-aminopyridine; C12 H17

O6 1-,C5 H7 N2 1+; G.Smith, R.C.Bott, U.D.Wermuth; Acta Crystallogr.,Sect.C:

Cryst.Struct.Commun., 56, 1505, 2000.

XELKOO 1,1,6,6-Tetraphenylhexa-2,4-diyne-1,6-diol bis(3-methylpyridine) clathrate; C30 H22

O2,2(C6 H7 N1); J.Bacsa, M.R.Caira, A.Jacobs, L.R.Nassimbeni, F.Toda; Crystal Engineering , $3,251,2000$.

XELKUU 1,1,6,6-Tetraphenylhexa-2,4-diyne-1,6-diol bis(pyridine) clathrate; C30 H22 O2,2(C6 H7

N1); J.Bacsa, M.R.Caira, A.Jacobs, L.R.Nassimbeni, F.Toda; Crystal Engineering , 3, 251, 2000.

XESPEQ Pyridinium 2,4-dinitrobenzoate; C5 H6 N1 1+,C7 H3 N2 O6 1-; L.Sobczyk, T.Lis, Z.Olejnik, I.Majerz; J.Mol.Struct., 552, 233, 2000.

XESPEQ01 Pyridinium 2,4-dinitrobenzoate; C5 H6 N1 1+,C7 H3 N2 O6 1-; L.Sobczyk, T.Lis, Z.Olejnik, I.Majerz; J.Mol.Struct., 552, 233, 2000.

XEYLOC 2,2'-Dihydroxy-1,1'-binaphthyl bis(2,4-lutidine) clathrate; C20 H14 O2,2(C7 H9 N1);

E.de Vries, L.R.Nassimbeni, Hong Su; Eur.J.Org.Chem., , 1887, 2001.

XEYMAP 2,2'-Dihydroxy-1,1'-binaphthyl 3,5-lutidine clathrate; C20 H14 O2,C7 H9 N1; E.de

Vries, L.R.Nassimbeni, Hong Su; Eur.J.Org.Chem., , 1887, 2001.

XICBAM pyridinium 2,4,6-trimercaptotriazine-1-ide 2,4,6-t rimercaptotriazine pyridine solvate; C5 H6 N1 1+,C3 H2 N3 S3 1-,2(C3 H3 N3 S3),2(C5 H5 N1); M.K.Krepps, S.Parkin, D.A.Atwood; Cryst.Growth Des., 1, 291, 2001.

XINNEN 1,10-Dinitro-2-oxo-guaia-3,5(6)-dien-13-oic acid pyridine solvate; C15 H18 N2 O7,C5 H5 N1; S.M.Adekenov, O.V.Alebastrov, V.A.Raldugin, I.Yu.Bagryanskaya, Yu.V.Gatilov, M.M.Shakirov, A.T.Kulyyasov, G.A.Tolstikov; Khim.Prir.Soedin(Russ.)(Chem.Nat.Compnd.), , 197, 2001.

XINRUH bis(2-Aminopyridinium) fumarate fumaric acid; 2(C5 H7 N2 1+),C4 H2 O4 2-,C4 H4 O4; A.Ballabh, D.R.Trivedi, P.Dastidar, E.Suresh; CrystEngComm , 4, 135, 2002. 
XINRUH01 bis(2-Aminopyridinium) fumarate fumaric acid; 2(C5 H7 N2 1+),C4 H2 O4 2-,C4 H4

O4; O.Buyukgungor, M.Odabasoglu, C.Albayrak, P.Lonnecke; Acta Crystallogr.,Sect.C: Cryst.Struct.Commun., 60, o470, 2004.

XINSES 2-Aminopyridinium hydrogen cyclobutane-1,1-dicarboxylate; C5 H7 N2 1+,C6 H7 O4 1-

; A.Ballabh, D.R.Trivedi, P.Dastidar, E.Suresh; CrystEngComm , 4, 135, 2002.

YABLAP 2,4-Dimethylpyridinium bis(4-bromobenzenesulfonyl)amide; C7 H10 N1 1+,C12 H8 Br2 N1 O4 S2 1-; V.Lozano, O.Moers, P.G.Jones, A.Blaschette; Z.Naturforsch.,BChem.Sci., 59, 661, 2004.

YEJSAH bis(4-Aminopyridinium) 2,5-dioxidobenzoquinone dihydrate; C6 H2 O4 2-,2(C5 H7 N2 1+),2(H2 O1); J.A.Cowan, J.A.K.Howard, M.A.Leech, I.D.Williams; Acta Crystallogr.,Sect.E: Struct.Rep.Online , 57, o563, 2001.

YEMJAB 2-Aminopyridinium 2-butynoate; C5 H7 N2 1+,C4 H3 O2 1-; K.A.Wheeler,

B.M.Foxman; Mol.Cryst.Liq.Cryst.Sci.Technol.,Sect.A , 240, 89, 1994.

YETLIS 3-Hydroxy-6-methylpyridinium hydrogen L-malate; C4 H5 O5 1-,C6 H8 N1 O1 1+;

C.B.Aakeroy, M.Nieuwenhuyzen; J.Am.Chem.Soc., 116, 10983, 1994.

YETLUE 3-Hydroxypyridinium hydrogen L-malate; C4 H5 O5 1-,C5 H6 N1 O1 1+; C.B.Aakeroy, M.Nieuwenhuyzen; J.Am.Chem.Soc., 116, 10983, 1994.

YIDKEB 4-Methylpyridinium 2,6-dichloro-4-nitrophenolate; C6 H8 N1 1+,C6 H2 Cl2 N1 O3 1-; I.Majerz, W.Sawka-Dobrowolska, L.Sobczyk; Pol.J.Chem., 67, 1657, 1993.

YINFAC 4-Methylpyridine trihydrate; C6 H7 N1,3(H2 O1); M.Born, D.Mootz, S.Schaefgen; Z.Naturforsch.,BChem.Sci., 50, 101, 1995.

YUTCIZ Cyclotetracatechylene pyridine methanol clathrate; C28 H24 O8,2(C5 H5 N1),2(C1 H4

O1); L.J.Barbour, J.W.Steed, J.L.Atwood; J.Chem.Soc.,Perkin Trans.2 , , 857, 1995.

ZOKSUN 4-N,N-Dimethylaminopyridinium pentachlorophenolate; C7 H11 N2 1+,C6 Cl5 O1 1-; I.Majerz, Z.Malarski, W.Sawka-Dobrowolska; J.Chem.Cryst., 25, 189, 1995.

ZUFDEJ 3-Cyano-4-methyl-6-phenylsulfonylamino-2-bis(phenylsulfonyl)amino-pyridine pyridine solvate; C25 H20 N4 O6 S3,C5 H5 N1; A.R.Katritzky, S.Rachwal, T.P.Smith, P.J.Steel; J.Heterocycl.Chem., 32, 979, 1995.

ZUWMAF 2-Ethylpyridinium 2,6-dichloro-4-nitrophenolate; C7 H10 N1 1+,C6 H2 Cl2 N1 O3 1-; I.Majerz, W.Sawka-Dobrowolska; J.Chem.Cryst., 26, 147, 1996. 